Mon. Not. R. Astron. Soc. 000, 田-16 (2001) Printed 22 October $2018 \quad$ (MN LATEX style file v2.2)

\title{
Cataclysmic Variables with Evolved Secondaries and the Progenitors of AM CVn Stars
}

\author{
Ph. Podsiadlowski ${ }^{1 \star}$, Z. $\operatorname{Han}^{2} \&$ S. Rappaport ${ }^{3}$ \\ 1 University of Oxford, Nuclear and Astrophysics Laboratory, Oxford, OX1 3RH \\ 2 Yunnan Observatory, National Astronomical Observatories, the Chinese Academy of Sciences, Kunming, 650011, China \\ 3 Department of Physics and Center for Space Research, Massachusetts Institute of Technology, Cambridge, MA 02139, USA
}

22 October 2018

\begin{abstract}
We present the results of a systematic study of cataclysmic variables (CVs) and related systems, combining detailed binary-population synthesis (BPS) models with a grid of 120 binary evolution sequences calculated with a Henyey-type stellar evolution code. In these sequences, we used 3 masses for the white dwarf $\left(0.6,0.8,1.0 \mathrm{M}_{\odot}\right)$ and seven masses for the donor star in the range of $0.6-1.4 \mathrm{M}_{\odot}$. The shortest orbital periods were chosen to have initially unevolved secondaries, and the longest orbital period for each secondary mass was taken to be just longer than the bifurcation period $(16-22 \mathrm{hr})$, beyond which systems evolve towards long orbital periods. These calculations show that systems which start with evolved secondaries near the end or just after their main-sequence phase become ultra-compact systems with periods as short as $\sim 7 \mathrm{~min}$. These systems are excellent candidates for AM CVn stars. Using a standard BPS code, we show how the properties of CVs at the beginning of mass transfer depend on the efficiency for common-envelope (CE) ejection and the efficiency of magnetic braking. In our standard model, where $\mathrm{CE}$ ejection is efficient, some 10 per cent of all CVs have initially evolved secondaries (with a central hydrogen abundance $X_{c}<0.4$ ) and ultimately become ultra-compact systems (implying a Galactic birthrate for AM CVn-like stars of $\sim 10^{-3} \mathrm{yr}^{-1}$ ). While these systems do not experience a period gap between 2 and $3 \mathrm{hr}$, their presence in the gap does not destroy its distinct appearance. Almost all $\mathrm{CVs}$ with orbital periods longer than $\sim 5 \mathrm{hr}$ are found to have initially evolved or relatively massive secondaries. We show that their distribution of effective temperatures is in good agreement with the distribution of spectral types obtained by Beuermann et al. (1998).
\end{abstract}

Key words: binaries: close - stars: AM CVn stars - stars: cataclysmic variables stars: white dwarfs - gravitation

\section{INTRODUCTION}

AM Canum Venaticorum (AM CVn) stars are ultra-compact binaries with orbital periods as short as $\sim 17 \mathrm{~min}$ (see Smak 1967 and Warner \& Robinson 1972 for the original identification). Because of the short orbital period, at least one of the binary components, and possibly both, is believed to be a degenerate dwarf (Paczyńksi 1967; Faulkner, Flannery \& Warner 1972). Because their evolution is driven by the loss of orbital angular momentum due to gravitational radiation, they have also been considered important sources of background gravitational radiation (Evans, Iben \& Smarr 1987; Hils, Bender \& Webbink 1990; Han \& Webbink 1999).
At present there are two popular models for the formation of AM CVn stars: (1) a double-degenerate model and (2) a model where the secondary is a semi-degenerate helium star. In the double-degenerate model (Tutukov \& Yungelson 1979; Nather, Robinson \& Stover 1981), AM CVn stars consist of two degenerate dwarfs that formed a close binary after experiencing one or two common-envelope phases (Paczyński 1976; Iben \& Tutukov 1984; Webbink 1984); the lighter component of the system (typically a He white dwarf) is now observed in the process of transferring mass to the more massive companion in a stable manner (for a detailed analysis of the stability of mass transfer of double degenerates, see Han \& Webbink 1999). In the second model, several phases of mass transfer first lead to the formation of a detached system consisting of a non-degenerate low-mass he-

\footnotetext{
^ E-mail: podsi@astro.ox.ac.uk
} 
Table 1. Orbital periods and model parameters for AM CVn stars

\begin{tabular}{|c|c|c|c|c|c|c|c|c|c|}
\hline \multirow[b]{2}{*}{ Name } & \multicolumn{3}{|c|}{ Observed Parameters } & \multicolumn{4}{|c|}{ Model Parameters } & \multicolumn{2}{|c|}{ Others } \\
\hline & $\begin{array}{l}\text { Period } \\
\text { (s) }\end{array}$ & $\begin{array}{c}m_{V} \\
(\mathrm{mag})\end{array}$ & Ref. & $\begin{array}{c}M_{2} \\
\left(\mathrm{M}_{\odot}\right)\end{array}$ & $\begin{array}{c}\log \dot{M} \\
\left(\mathrm{M}_{\odot} \mathrm{yr}^{-1}\right)\end{array}$ & $\begin{array}{c}\log (P /|\dot{P}|) \\
(\mathrm{yr})\end{array}$ & $X$ & $\begin{array}{c}M_{2}(\mathrm{DD}) \\
\left(\mathrm{M}_{\odot}\right)\end{array}$ & $\begin{array}{c}M_{2}(\mathrm{He}) \\
\left(\mathrm{M}_{\odot}\right)\end{array}$ \\
\hline AM CVn & 1029 & $14.1-1.42$ & 1 & $\begin{array}{l}0.077_{-0.002}^{+0.010} \\
0.065_{-0.002}^{+0.009}\end{array}$ & $\begin{array}{l}-8.8_{-0.0}^{+0.1} \\
-8.7_{-0.0}^{+0.1}\end{array}$ & $\begin{array}{l}7.9_{-0.4}^{+0.3} \\
8.1_{-0.3}^{+0.4}\end{array}$ & $\begin{array}{l}0.03_{-0.00}^{+0.06} \\
0.01_{-0.01}^{+0.02}\end{array}$ & 0.033 & 0.114 \\
\hline HP Lib & 1119 & 13.6 & 2 & $\begin{array}{l}0.081_{-0.003}^{+0.006} \\
0.061_{-0.003}^{+0.006}\end{array}$ & $\begin{array}{l}-8.9_{-0.0}^{+0.0} \\
-8.9_{-0.0}^{+0.1}\end{array}$ & $\begin{array}{l}7.9_{-0.1}^{+0.1} \\
8.2_{-0.4}^{+0.5}\end{array}$ & $\begin{array}{l}0.11_{-0.01}^{+0.01} \\
0.01_{-0.01}^{+0.01}\end{array}$ & 0.030 & 0.099 \\
\hline CR Boo & 1471 & $13.0-18.0$ & 3 & $\begin{array}{l}0.096_{-0.002}^{+0.001} \\
0.047_{-0.003}^{+0.004}\end{array}$ & $\begin{array}{l}-9.3_{-0.0}^{+0.0} \\
-9.5_{-0.1}^{+0.1}\end{array}$ & $\begin{array}{l}7.7_{-0.0}^{+0.4} \\
8.6_{-0.5}^{+0.5}\end{array}$ & $\begin{array}{l}0.19_{-0.00}^{+0.01} \\
0.02_{-0.02}^{+0.01}\end{array}$ & 0.021 & 0.062 \\
\hline V803 Cen & 1611 & $13.2-17.4$ & 2 & $\begin{array}{r}0.099_{-0.001}^{+0.003} \\
0.043_{-0.004}^{+0.004}\end{array}$ & $\begin{array}{l}-9.3_{-0.0}^{+0.0} \\
-9.6_{-0.1}^{+0.1}\end{array}$ & $\begin{array}{l}7.7_{-0.1}^{+0.0} \\
8.7_{-0.4}^{+0.5}\end{array}$ & $\begin{array}{l}0.21_{-0.00}^{+0.00} \\
0.03_{-0.03}^{+0.01}\end{array}$ & 0.019 & 0.054 \\
\hline CP Eri & 1724 & $16.5-19.7$ & 2 & $\begin{array}{l}0.100_{-0.021}^{+0.003} \\
0.040_{-0.004}^{+0.005}\end{array}$ & $\begin{array}{r}-9.4_{-0.0}^{+0.0} \\
-9.8_{-0.1}^{+0.1}\end{array}$ & $\begin{array}{l}7.8_{-0.1}^{+0.8} \\
8.7_{-0.4}^{+0.5}\end{array}$ & $\begin{array}{r}0.22_{-0.12}^{+0.00} \\
0.03_{-0.03}^{+0.01}\end{array}$ & 0.017 & 0.048 \\
\hline GP Com & 2970 & $15.7-16.0$ & 2 & $\begin{array}{l}0.037_{-0.003}^{+0.022} \\
0.031_{-0.009}^{+0.008}\end{array}$ & $\begin{array}{l}-10.7_{-0.1}^{+0.3} \\
-10.7_{-0.3}^{+0.2}\end{array}$ & $\begin{array}{l}9.9_{-0.6}^{+0.2} \\
9.5_{-0.4}^{+0.6}\end{array}$ & $\begin{array}{l}0.23_{-0.07}^{+0.02} \\
0.20_{-0.20}^{+0.05}\end{array}$ & 0.008 & 0.019 \\
\hline
\end{tabular}

Note. $-m_{V}$ : visual magnitude; $M_{2}$ : mass of secondary; $\dot{M}$ : mass-transfer rate; $(P /|\dot{P}|)$ : timescale for orbital period change; $X$ : surface hydrogen abundance. Most model parameters are from the present study, where the values in the first row are for systems before the period minimum (i.e. when their orbital period decreases) and the values in the second row are for systems after the period minimum (i.e. when their orbital period increases). The alternative values for $M_{2}$ listed under 'Others' were taken from NPVY (as well as the observational parameters and references); the column marked 'DD' assumes a double-degenerate model and the column marked 'He' assumes a semi-degenerate helium-star model.

References. - (1) Patterson et al. (1993), (2) Warner (1995), (3) Provencal et al. (1997).

lium star (typically $\sim 0.4-0.6 \mathrm{M}_{\odot}$ ) and a white-dwarf companion. If the orbital period of this system is short enough, angular-momentum loss due to gravitational radiation will cause the orbit to decay until the helium star fills its Roche lobe and starts to transfer mass (at an orbital period $\lesssim 1 \mathrm{hr}$ ); the system will now appear as an AM CVn star (Savonije, de Kool \& van den Heuvel 1986; Iben \& Tutukov 1991). The orbital period will decrease further, reaching a minimum period of $\sim 10 \mathrm{~min}$, and then start to increase again (while the secondary becomes semi-degenerate).

Nelemans et al. (2001a; NPVY) have recently published a thorough binary-population synthesis (BPS) study of AM CVn stars and found that both of these evolutionary channels could be of comparable importance; this depends, however, on many uncertain factors in the modelling. (We refer to this paper and Warner [1995] for excellent reviews of AM CVn stars and further references.) In Table 1 we list the observational properties of 6 known AM CVn stars, taken directly from NPVY, and some model parameters from NPVY and the present investigation $(\S 3)$ ].

In addition to these two channels there is a third chan-

1 NPVY also list RX J1914+24 as a further AM CVn candidate. In this system, Cropper et al. (1998) have observed a period of $569 \mathrm{~s}$, which they interpret as the spin period of a magnetic white dwarf; they further suggest that the spin of the white dwarf may be magnetically locked to the orbit, in which case this period would also constitute the orbital period of the system, making it the binary with the shortest known orbital period. Since we consider this interpretation uncertain at the present time, we have not listed this system, but note that the model described in $\S 2$ can explain systems with periods as short as $\sim 7 \mathrm{~min}$. nel that leads to the formation of ultra-compact systems, which is well established in the context of ultra-compact systems containing a neutron star (Tutukov et al. 1987; Fedorova \& Ergma 1989; Podsiadlowski, Rappaport \& Pfahl 2001 [PRP]). It requires only that a normal H-rich star $\left(\sim 1 \mathrm{M}_{\odot}\right)$ starts to transfer mass near the end of or just after hydrogen core burning. Such systems become ultra-compact binaries with periods as short as $\sim 10 \mathrm{~min}$ where the secondary is initially non-degenerate and hydrogen-rich, but increasingly becomes degenerate and helium-rich during its evolution. NPVY dismissed this channel as one of low probability. However, in a recent study of low-/intermediate-mass X-ray binaries (LMXBs/IMXBs), PRP found that the initial period range that leads to ultra-compact systems for a secondary with an initial mass of $1 \mathrm{M}_{\odot}$ is quite large, 13 to 18 $\mathrm{hr}$, and suggested that this is the reason why ultra-compact LMXBs are so common in globular clusters. In addition, this alternative channel depends on relatively few uncertainties in the theoretical modelling of the binary evolution. It is one of the purposes of this paper to demonstrate that this channel indeed provides a viable alternative channel for the formation of AM CVn stars.

During their early evolution, such systems will appear as cataclysmic variables (CVs) with evolved secondaries. Recently, Beuermann et al. (1998) and Smith \& Dhillon (1998) have shown that, for orbital periods longer than $\sim 5 \mathrm{hr}$, there is a large spread in the observed spectral types of the secondaries in CVs at a given orbital period and suggested that a large fraction of these systems had companions with evolved secondaries. These evolved systems are excellent candidates for the progenitors of AM CVn systems. However, these observations also pose an immediate problem for the standard 
explanation of the period gap in CVs between 2 and $3 \mathrm{hr}$, the disrupted-magnetic braking model (Rappaport, Verbunt \& Joss 1983; Spruit \& Ritter 1983). In this model, magnetic braking dramatically decreases when the secondary becomes fully convective (at an orbital period of $\sim 3 \mathrm{hr}$ ), allowing it to relax to thermal equilibrium and to become detached. Mass transfer starts again once angular-momentum loss due to gravitational radiation has brought the secondary back into contact which occurs at an orbital period of $\sim 2 \mathrm{hr}$ (for detailed studies see Kolb 1993; Howell, Nelson \& Rappaport $2001[\mathrm{HNR}]$ ). However, it is well established that CVs that start with only a slightly evolved secondary experience a gap at shorter orbital periods or no period gap at all (Pylyser $\&$ Savonije 1989; PRP and $\S 2$ ). Hence if the majority of all CVs had an evolved companion initially, this would challenge the standard explanation for the period gap. However, as we will show in this contribution there is no serious conflict between the existence of evolved systems and the period gap, even for quite standard assumptions?.

In $\S 2$ of this paper we briefly summarize our binary stellar evolution calculations and present detailed calculations to illustrate the formation of ultra-compact, AM CVn-like systems. In $\S 3$ we use a large grid of these binary evolution sequences and integrate them into a standard binary population synthesis code to simulate the main properties of CVs and related objects. In $\S 4$ we discuss the implications of these results for ultra-compact systems (like AM CVn's) and CVs with evolved secondaries and compare our simulations with observations.

\section{BINARY STELLAR EVOLUTION CALCULATIONS}

All binary evolution calculations were carried out with a standard Henyey-type stellar evolution code (Kippenhahn, Weigert \& Hofmeister 1967), using solar metallicity $(Z=$ 0.02 ), up-to-date opacities and a mixing-length parameter $\alpha=2$ (for a detailed description see PRP). We include angular-momentum loss due to gravitational radiation and magnetic braking. For the latter we use the formalism of Rappaport, Verbunt \& Joss (1983; their eq. 36 with $\gamma=4)$, which is based on the magnetic-braking law of Verbunt \& Zwaan (1981). We only include magnetic braking, if the secondary (the mass-losing component) has a sizeable convective envelope, and assume that it stops abruptly when the secondary becomes fully convective (Rappaport et al. 1983; Spruit \& Ritter 1983). We further assume that mass transfer is completely non-conservative, i.e. that all the mass lost from the secondary is ejected from the system (e.g. in the form of nova explosions or a disc wind), where the matter

2 Baraffe \& Kolb (2000) attempted to solve this apparent conflict by proposing that evolved systems have much higher masstransfer rates than obtained in standard binary evolutionary calculations and 'bounced' before reaching the period gap, i.e. never reach orbital periods $\lesssim 3 \mathrm{hr}$. However, this constitutes a significant departure from the standard model for the evolution of CVs and would in our view compromise some of the fundamental assumptions of this model, that has been so successful in explaining key observational features of CVs (see e.g. Kolb 1983; HNR]). that is lost from the system carries with it the specific orbital angular momentum of the white dwarf.

In Figure 1 we present the results of four binary sequences for a white-dwarf accretor with mass $M_{1}=0.6 \mathrm{M}_{\odot}$ and a normal donor star with an initial mass $M_{2}=1 \mathrm{M}_{\odot}$ for different evolutionary phases of the donor at the beginning of mass transfer: unevolved donor (solid curves), evolved donor with a central hydrogen abundance of $X_{c}=0.10$ (dashed curves), evolved donor with hydrogen-exhausted cores of mass $M_{c}=0.037 \mathrm{M}_{\odot}$ (dot-dashed curves) and $M_{c}=0.063 \mathrm{M}_{\odot}$ (dot-dot-dot-dashed curves), respectively. (This figure is analogous to fig. 16 in PRP.)

First consider the sequence with an initially unevolved secondary (dashed curves). Since the secondary is initially significantly more massive than the white-dwarf primary, it cannot remain in near thermal equilibrium, and mass transfer initially occurs on a thermal timescale, reaching a maximum mass-transfer rate of $\sim 5 \times 10^{-8} \mathrm{M}_{\odot} \mathrm{yr}^{-1}$. Even after the mass ratio has been reversed, it cannot relax to thermal equilibrium since the timescale for magnetic braking, the dominant mechanism driving mass transfer at this stage, is comparable to the Kelvin-Helmholtz timescale of the secondary $\left(\sim 2 \times 10^{8} \mathrm{yr}\right)$. This changes abruptly when the secondary becomes fully convective and magnetic braking is assumed to become ineffective (at an orbital period of $3.07 \mathrm{hr}$ in this example). Now it can relax and shrink to its equilibrium radius, associated with a kink in the HertzsprungRussell (H-R) diagram (left panel in Fig. 1). The system remains detached until angular momentum loss due to gravitational radiation brings the secondary back into contact with its Roche lobe (at an orbital period of $2.45 \mathrm{hr}$ ). Gravitational radiation, which is the dominant mass-transfer driving mechanism now, continues to drive the orbital evolution of the system, but at a lower rate than magnetic braking did, leading to a lower mass-transfer rate below the period gap. The orbital period decreases until it reaches a minimum at $75 \mathrm{~min}$ and then starts to increase again (while the secondary is becoming increasingly degenerate).

There are several points to note about this sequence. First, the period gap between 2.45 and $3.07 \mathrm{hr}$ in this sequence (as well as in all other sequences with unevolved secondaries) is somewhat smaller than the period gap found in other studies of CVs (e.g. Kolb 1993; HNR) which were specifically designed to explain the observed period gap between 2 and 3 hr (Ritter \& Kolb 1998). This is not surprising since we did not calibrate our magnetic-braking law to reproduce the period gap. The fact that our gap is too small implies that our model somewhat underestimates the amount of magnetic braking just above the gap. Second, the period minimum found in our sequences is somewhat higher (at $75 \mathrm{~min}$ ) than the period minimum obtained in other recent studies of CVs ( $65 \mathrm{~min}$, Kolb \& Baraffe 1999; HNR). Since these other investigations use more sophisticated equations of state, more appropriate in the regime of very-low-mass secondaries near the period minimum, their quoted values are likely to be more realistic and should be preferred. Despite these limitations, we do not believe that these differences will affect any of the main conclusions in this paper.

Consider now the dashed sequence, where the secondary starts to transfer mass when it is already quite evolved, and its central hydrogen abundance (by mass) has decreased to $X_{c}=0.10$. In this case, the secondary becomes fully con- 

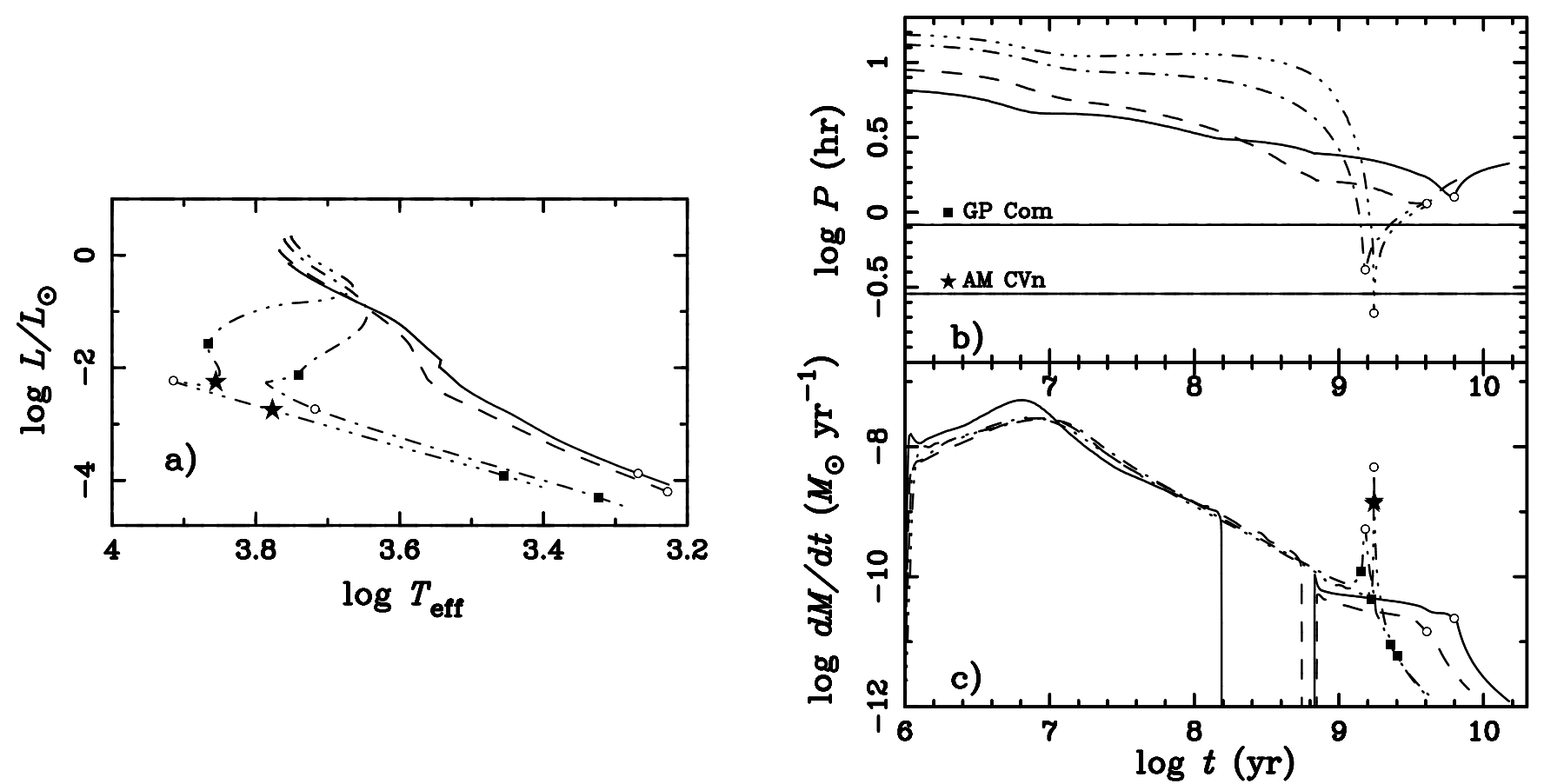

Figure 1. Selected binary sequences illustrating the formation of AM CVn systems. All systems initially consist of a white dwarf of $0.6 \mathrm{M}_{\odot}$ and a normal-star companion of $1 \mathrm{M}_{\odot}$ for different initial orbital periods (line styles from short to long initial period: solid, dashed, dot-dashed, dot-dot-dot-dashed). The individual panels show the evolutionary tracks in the H-R diagram (left), the evolution of orbital period (top right) and mass-transfer rate (bottom right) as a function of time since the beginning of mass transfer. The open circles show where the systems in the individual sequences reach their period minimums. Other symbols indicate when the systems pass through the periods of two representative AM CVn systems (squares: GP Com, stars: AM CVn).

vective at a much lower mass $\left(0.14 \mathrm{M}_{\odot}\right)$, implying a much shorter orbital period when the system becomes detached. Since the magnetic-braking timescale is much longer at this shorter period, the secondary is less out of thermal equilibrium and less oversized relative to is equilibrium radius. As a consequence, the system experiences only a very short period gap between 1.30 and $1.45 \mathrm{hr}$. We find quite generally that for systems to experience a significant period gap between 2 and $3 \mathrm{hr}$, they have to be relatively unevolved initially; typically the central hydrogen abundance at the beginning of mass transfer has to be larger than $\sim 0.40$ (see Table A1). After gravitational radiation has brought the system back into contact, the subsequent evolution is similar to the previous case, but reaches a period minimum at a substantially lower orbital period of $55 \mathrm{~min}$.

In the two other cases, where the secondaries already have hydrogen-exhausted cores of $0.037 \mathrm{M}_{\odot}$ and $0.063 \mathrm{M}_{\odot}$ at the beginning of mass transfer, respectively, there is no period gap, and the secondaries transform themselves into essentially pure helium white dwarfs (with some traces of hydrogen, 5 and 4 per cent left initially). These systems reach extremely short orbital periods of 22 and $11 \mathrm{~min}$, respectively. Note that near the minimum period (open circles in Fig. 1), the secondaries are still relatively luminous and hot, but then cool rapidly and join the appropriate white-dwarf cooling curve. Since the gravitational radiation timescale becomes very short at these short orbital periods, the masstransfer rate shows a sharp spike near the period minimum. Both of these sequences pass through the period range of many AM CVn stars, once when the orbital period is decreasing and once after the period minimum (very similar to the behaviour of the analogous ultra-compact systems with neutron-star primaries; see Fedorova \& Ergma 1989 and PRP). One possible way of distinguishing between these two passages is that the secondaries in systems before the period minimum have larger amounts of hydrogen left in their envelopes. Systems that have hydrogen-exhausted cores at the beginning of mass transfer transform themselves into essentially pure helium white dwarfs near the period minimum (see the column marked ' $X$ ' in Table 1 and $\S 4$ ). In addition, the rates of change in the orbital period will be of opposite sign.

In order to investigate this channel for ultra-compact AM CVn-like systems more systematically, we have carried out a large number of binary evolution calculations, where we varied the initial masses of both components and the evolutionary phase at the beginning of mass transfer. Altogether we performed 120 such calculations for three masses of the primary $\left(M_{1}=0.6,0.8\right.$ and $\left.1.0 \mathrm{M}_{\odot}\right)$ and seven masses of the secondary $\left(M_{2}=0.6,0.8,0.9,1.0,1.1,1.2\right.$ and $\left.1.4 \mathrm{M}_{\odot}\right)$. The shortest orbital period for each combination of masses was chosen so that the secondary filled its Roche lobe on the zero-age main sequence (i.e. when it was completely unevolved). The longest orbital period was determined so that it was slightly larger than the bifurcation period above which systems evolve towards longer orbital periods rather than shorter orbital periods (see e.g. Pylyser \& Savonije 1989 and PRP). The value of the bifurcation period was found to vary from $\sim 16 \mathrm{hr}$ for the sequences with a $1 \mathrm{M}_{\odot}$ secondary to $\sim 22 \mathrm{hr}$ for the most massive sequences. In cases where the main-sequence lifetime was longer than $12 \times 10^{10}$ yr (for the lower-mass secondaries), the largest period was chosen 
by the constraint that the secondary can evolve to fill its Roche lobe in less than $12 \times 10^{10} \mathrm{yr}$.

Some of the key characteristics of each sequence are given in the appendix in Table A1. Our grid of models covers a wide range of evolutionary behaviours, from systems that are exclusively driven by angular-momentum loss due to magnetic braking and gravitational radiation (classical CVs) to systems that experience rapid mass transfer on a thermal timescale and would appear as supersoft X-ray sources (see e.g. Rappaport, Di Stefano \& Smith 1994; Langer et al. 2000; King et al. 2001). We note that in all calculated sequences, mass transfer was dynamically stable at all times. The only sequence that was close to dynamical instability was the sequence with a $0.6 \mathrm{M}_{\odot}$ white dwarf and an initially unevolved $1.4 \mathrm{M}_{\odot}$ secondary.

\section{BINARY POPULATION SYNTHESIS}

The determination of the statistical properties of CVs and AM CVn stars requires a binary population synthesis (BPS) study, which starts with a sample of primordial binaries with given distributions of component masses and orbital periods, follows the various evolutionary paths encountered and derives the statistical properties of the systems of interest. The BPS method utilized in this investigation differs from most previous studies of this kind in so far as we use detailed binary evolution sequences to model the evolutionary phase of interest, the CV phase, instead of relying on simplified prescriptions for the evolution. This allows us to also include phases of mass transfer that occur on a thermal timescale and phases where the chemical composition of the mass donor changes, which cannot be reliably treated with simplified prescriptions (unlike the case of classical CVs; see e.g. HNR). More specifically, we directly link the grid of 120 binary sequences described in the previous section with a standard BPS code: we first use the BPS code to determine the distributions of masses and orbital periods at the beginning of the $\mathrm{CV}$ phase and to assign relative weights to the individual sequences, and then we use these properly weighted sequences to construct the statistical properties of CVs.

\subsection{The binary population synthesis code}

The BPS code we use in this study was originally developed by Han (1995) and is described in detail in Han, Podsiadlowski \& Eggleton (1995; HPE). Since then the code has been continuously revised and updated, as described in Han et al. (2001). Here we restrict ourselves to listing some of the main assumptions as relevant for the present investigation.

The code uses a standard Monte-Carlo technique, where we typically simulate the evolution of $10^{7}$ systems. For the initial distributions, we assume a Miller-Scalo distribution for the primary (Miller \& Scalo 1979), take the mass-ratio distribution to be flat, and adopt a distribution of initial orbital separation that is flat in $\log a$. We further assume that the star-formation rate is constant over the age of the Galaxy (taken to be $12 \mathrm{Gyr}$ ), forming one binary system with a primary mass larger than $0.8 \mathrm{M}_{\odot}$ per year in the Galaxy (this corresponds to an integrated Galactic star-formation rate by mass of $\left.3.5 \mathrm{M}_{\odot} \mathrm{yr}^{-1}\right)$. This star-formation rate implies the formation of $\sim 1$ white dwarf (either single or in a binary) per year in the Galaxy.

All of these assumptions are fairly standard. Where our method differs significantly from most other BPS studies is in the treatment of the common-envelope and the spiral-in phase (the conditions for the occurrence of dynamical mass transfer are described in detail in Han et al. 2001). As in most other studies, we assume that the common envelope is ejected when the change in orbital energy, $\Delta E_{\text {orb }}$, times some factor, $\alpha_{\mathrm{CE}}$, exceeds the binding energy of the envelope. Unlike most other studies, however, we do not use an analytic formula for the binding energy, but use the values obtained from detailed stellar calculations and also include a contribution from the thermal energy of the envelope (see HPE for details and also Dewi \& Tauris 2000). Our envelope ejection criterion is written as

$\alpha_{\mathrm{CE}}\left|\Delta E_{\text {orb }}\right|>\left|E_{\mathrm{gr}}+\alpha_{\mathrm{th}} E_{\mathrm{th}}\right|$,

where $E_{\text {gr }}$ is the gravitational potential energy of the envelope and $E_{\text {th }}$ its thermal energy, which in particular includes the ionization energy. The inclusion of the ionization energy can change the orbital-period distribution immediately after the ejection of the common envelope (CE) quite dramatically, since for evolved stars on the asymptotic-giant branch (AGB), the total energy of the envelope becomes small and ultimately zero if the ionization energy is included (see Han, Podsiadlowski \& Eggleton 1994). If this ionization energy can be extracted in the CE ejection process, very little orbital shrinking is required to provide enough energy to eject the loosely bound envelopes on the AGB, leading to relatively long post-CE orbital periods. To account for this possibility, we therefore use two parameters rather than one, $\alpha_{\mathrm{CE}}$ and $\alpha_{\mathrm{th}}$, in our definition of the CE ejection criterion. In the following, we will use two sets of parameters: a set for efficient $\mathrm{CE}$ ejection (with $\alpha_{\mathrm{CE}}=\alpha_{\mathrm{th}}=1$ ), and a less efficient one (with $\alpha_{\mathrm{CE}}=0.5, \alpha_{\mathrm{th}}=0$ ). The first set assumes the maximum CE-ejection efficiency consistent with energy conservation and leads to a post-CE distribution of orbital periods extending to $\sim 420 \mathrm{~d}$, while the second produces a distribution that is limited to less than $\sim 6 \mathrm{~d}$ (see e.g. figure 4 in $\mathrm{HPE}$ ). We generally favour the more efficient ejection criterion in order to be able to explain certain systems containing a white dwarf (some symbiotic binaries and barium stars; see the discussions in HPE and Han et al. 1995), which have orbital periods that are relatively long ( $\gtrsim 100 \mathrm{~d}$ ), but appear to be too short to have avoided a CE phase altogether.

\subsection{The distributions at the beginning of mass transfer}

The progenitors of CVs are generally believed to be relatively wide systems with orbital periods of several years that experience a $\mathrm{CE}$ and spiral-in phase, leaving a tight binary with a period of less than a few days after the envelope has been ejected (Paczyński 1976). In most cases, the system will be detached immediately after the CE phase, but will come back into contact due to either of two complementary effects: the first is the orbital shrinking due to the loss of orbital angular momentum caused by magnetic braking and gravitational radiation; the second is the expansion of the secondary due to it own nuclear evolution (for stars that are 


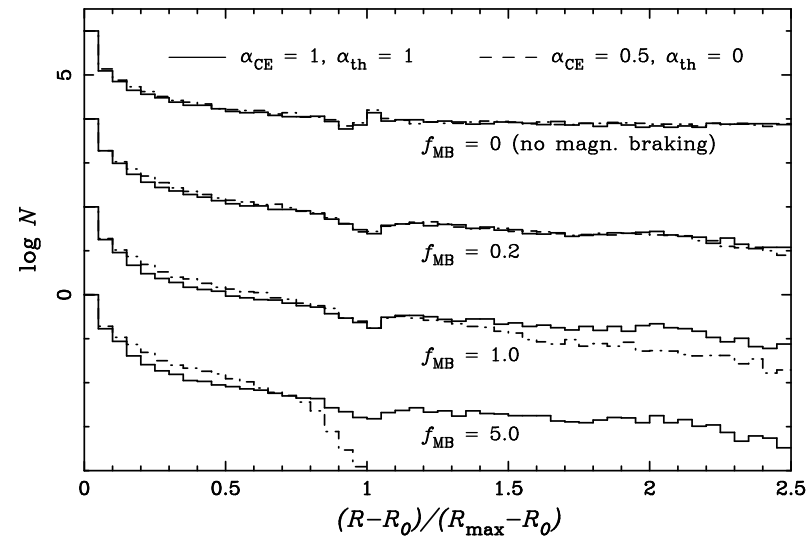

Figure 2. Number distribution of the evolution parameter $r=$ $\left(R-R_{0}\right) /\left(R_{\max }-R_{0}\right)$ for different BPS models and assumptions about the efficiency of magnetic braking, where $R$ is the radius of the secondary at the beginning of mass transfer, $R_{0}$ and $R_{\max }$ are the radii of a star of the same mass at the beginning of hydrogen burning and at the point of hydrogen exhaustion, respectively. Solid histograms are for models with efficient common-envelope (CE) ejection $\left(\alpha_{\mathrm{CE}}=1\right.$ and $\left.\alpha_{\mathrm{th}}=1\right)$, dot-dashed histograms for models with less efficient CE ejection $\left(\alpha_{\mathrm{CE}}=0.5\right.$ and $\left.\alpha_{\mathrm{th}}=0\right)$. The histograms for different efficiencies of magnetic braking (as indicated) have been shifted by two orders of magnitude relative to each other for clarity.

massive enough that they evolve appreciably in a Hubble time). It is the balancing of these two effects that determines the evolutionary state of the secondary when it starts to transfer mass. As already discussed in $\S 2$, the standard explanation for the period gap requires that most secondaries have to be relatively unevolved at the beginning of mass transfer, which implies that for the majority of stars the timescale for angular-momentum losses has to be shorter than the evolutionary timescale of the secondary. This also has the somewhat unfortunate consequence that the distribution of the evolutionary states of the secondaries at the beginning of mass transfer is rather sensitive to the assumed strength of magnetic braking, which is still rather uncertain.

In Figure 2 we illustrate how the CE criterion and the assumptions concerning the efficiency of magnetic braking affect the distribution of evolutionary states of the secondary at the beginning of mass transfer. For this purpose we have defined an evolution parameter $r$ as

$r=\frac{R-R_{0}}{R_{\max }-R_{0}}$

where $R$ is the radius of the secondary at the beginning of mass transfer, and $R_{0}$ and $R_{\max }$ are the radius of the secondary on the zero-age main sequence and at the point of core hydrogen exhaustion, respectively; i.e. for a completely unevolved star $r=0$, and for a star at the end of its main-sequence phase $r=1$. Figure 2 shows histograms of the evolution parameter for models with efficient and inefficient $\mathrm{CE}$ ejection, respectively, and for different efficiencies of magnetic braking, $f_{\mathrm{MB}}=0,0.2,1,5$, where we scaled the efficiency with the standard magnetic-braking description adopted in our binary evolutionary sequences (see $\S 2$ ). Note that in these distributions we did not include systems with He white dwarf primaries, since they are not represented in our grid of evolutionary sequences (although many of these

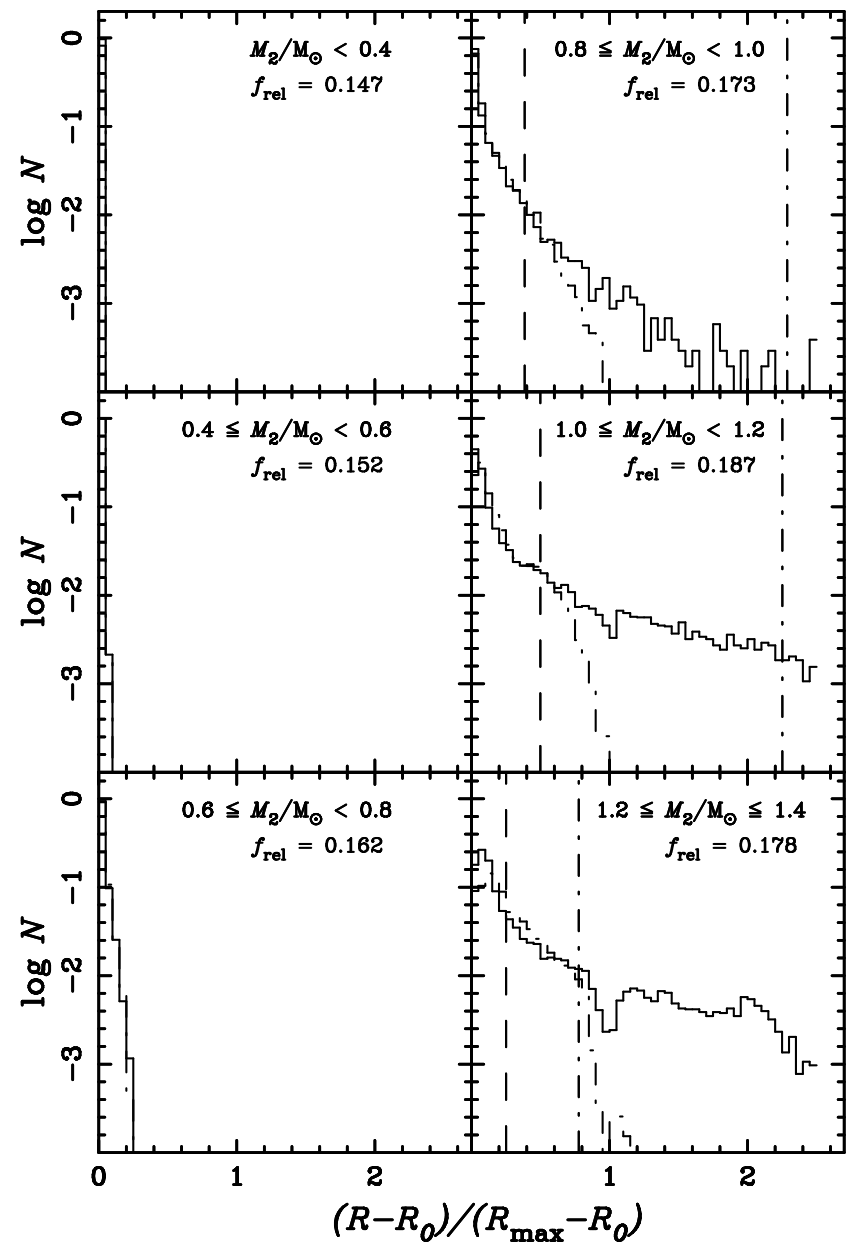

Figure 3. Number distributions as a function of evolution parameter (see Fig. 2) for CVs at the start of mass transfer for different mass ranges for the secondary, $M_{2}$, (as indicated in the individual panels). The solid histograms give the distributions for a model with efficient common-envelope (CE) ejection and standard magnetic braking (model 1), while the dashed histograms show the distributions for inefficient $\mathrm{CE}$ ejection and magnetic braking enhanced by a factor of 5 (model 8 ). The relative weights of the different mass ranges are denoted as $f_{\text {rel }}$ in each panel (for model 1 only). The vertical dashed and dot-dashed curves in the right panels indicate the range of evolution parameter in which systems may become AM CVn stars.

will, of course, also contribute to the CV population, see e.g. Politano 1996; HNR).

In all cases, the distributions are sharply peaked around $r=0$, i.e. are dominated by unevolved systems, as required in the standard explanation for the period gap, and show a tail extending towards long evolution parameter. If no magnetic braking is taken into account $\left(f_{\mathrm{MB}}=0\right)$, the distribution with efficient and inefficient $\mathrm{CE}$ ejection (the dashed and dot-dashed histograms at the top) are almost identical. As the efficiency of magnetic braking is increased, the tail with evolved systems decreases, and the distribution becomes even more concentrated around $r=0$, simply because magnetic braking brings an increasing number of systems into contact before they had time to evolve appreciably. The distributions with efficient and inefficient $\mathrm{CE}$ ejection also start to diverge. In the most extreme case, where magnetic 
Table 2. Galactic birth rates (per year) for CVs, ultra-compact CVs and CVs with evolved secondaries.

\begin{tabular}{|c|c|c|c|c|c|c|c|c|}
\hline \multirow[b]{2}{*}{$f_{\mathrm{MB}}$} & \multirow[b]{2}{*}{$\alpha_{\mathrm{CE}}$} & \multirow[b]{2}{*}{$\alpha_{\text {th }}$} & \multirow[b]{2}{*}{ total } & \multicolumn{3}{|c|}{$P_{\min }$} & \multirow[b]{2}{*}{$X_{c}<0.4$} & \multirow[b]{2}{*}{ model } \\
\hline & & & & $<25 \min$ & $<45 \min$ & $<70 \min$ & & \\
\hline \multirow[t]{2}{*}{1} & 1 & 1 & 6.0E-03 & $6.4 \mathrm{E}-05$ & $1.2 \mathrm{E}-04$ & $6.6 \mathrm{E}-04$ & $5.8 \mathrm{E}-04$ & (1) \\
\hline & 0.5 & 0 & $9.4 \mathrm{E}-03$ & $7.7 \mathrm{E}-05$ & $1.8 \mathrm{E}-04$ & $1.4 \mathrm{E}-03$ & $1.1 \mathrm{E}-03$ & $(2)$ \\
\hline \multirow[t]{2}{*}{0} & 1 & 1 & $2.2 \mathrm{E}-03$ & $1.1 \mathrm{E}-04$ & $1.5 \mathrm{E}-04$ & $4.6 \mathrm{E}-04$ & $4.8 \mathrm{E}-04$ & (3) \\
\hline & 0.5 & 0 & 4.1E-03 & $1.9 \mathrm{E}-04$ & $2.7 \mathrm{E}-04$ & 8.7E-04 & $8.8 \mathrm{E}-04$ & (4) \\
\hline \multirow[t]{2}{*}{0.2} & 1 & 1 & 4.7E-03 & $6.8 \mathrm{E}-05$ & $1.2 \mathrm{E}-04$ & $6.4 \mathrm{E}-04$ & $5.7 \mathrm{E}-04$ & (5) \\
\hline & 0.5 & 0 & $8.0 \mathrm{E}-03$ & $1.1 \mathrm{E}-04$ & $2.1 \mathrm{E}-04$ & $1.3 \mathrm{E}-03$ & $1.0 \mathrm{E}-03$ & (6) \\
\hline \multirow[t]{2}{*}{5} & 1 & 1 & 7.1E-03 & $6.4 \mathrm{E}-05$ & $1.2 \mathrm{E}-04$ & $6.8 \mathrm{E}-04$ & $5.6 \mathrm{E}-04$ & (7) \\
\hline & 0.5 & 0 & $1.0 \mathrm{E}-02$ & $1.4 \mathrm{E}-05$ & $6.8 \mathrm{E}-05$ & $1.3 \mathrm{E}-03$ & $9.4 \mathrm{E}-04$ & (8) \\
\hline 1 & 1 & 1 & $6.1 \mathrm{E}-03$ & $7.0 \mathrm{E}-05$ & $1.3 \mathrm{E}-04$ & $6.8 \mathrm{E}-04$ & $6.0 \mathrm{E}-04$ & $\left(1^{\prime}\right)$ \\
\hline 1 & 1 & 1 & 7.0E-03 & 9.0E-05 & $1.5 \mathrm{E}-04$ & $9.2 \mathrm{E}-04$ & $8.1 \mathrm{E}-04$ & $\left(1^{*}\right)$ \\
\hline
\end{tabular}

Note. - $f_{\mathrm{MB}}$ : efficiency of magnetic braking; $\alpha_{\mathrm{CE}}, \alpha_{\mathrm{th}}$ : common-envelope efficiency parameters; $P_{\min }$ : period minimum; $X_{c}$ : initial central hydrogen abundance of the secondary. Models (1) to (8) assume a Galactic age of $12 \mathrm{Gyr}$, model (1') of 15 Gyr. Model $\left(1^{*}\right)$ is a model similar to model (1) for a metallicity $Z=0.001$. Note that CVs with He white dwarfs were not included in these rates.

braking was assumed to be enhanced by a factor of 5 , there are no systems with $r>1$ for the model with inefficient $\mathrm{CE}$ ejection (the dot-dashed histogram at the bottom). The reason is that in this model, the post-CE orbital period distribution has a cut-off for periods above $\sim 6 \mathrm{~d}$, and magnetic braking is efficient enough to bring these systems into contact before any secondary (with $M_{2} \leq 1.4 \mathrm{M}_{\odot}$ ) had time to evolve off the main sequence.

To further illustrate the dependence of the distributions of evolution parameter on the mass of the secondary, we divided the distribution into different mass bins (see Fig. 3), both for our standard reference model (with $\alpha_{\mathrm{CE}}=\alpha_{\mathrm{th}}=$ $\left.f_{\mathrm{MB}}=1\right)$ and the extreme model with $\alpha_{\mathrm{CE}}=0.5, \alpha_{\mathrm{th}}=0$, $f_{\mathrm{MB}}=5$. For masses less than $0.8 \mathrm{M}_{\odot}$, all systems are relatively unevolved at the beginning of mass transfer, simply because their masses are too low for them to evolve appreciably in $12 \mathrm{Gyr}$, our assumed age for the Galaxy. These are the systems that have dominated in previous population synthesis studies of CVs (see de Kool 1992; Shafter 1991; Kolb 1993; Politano 1996; HNR). On the other hand, for more massive secondaries, the distributions contain a substantial fraction of evolved systems. In the panels on the right, the vertical dashed line indicates the approximate value for the evolution parameter (for each mass bin) above which systems will evolve towards ultra-short periods. The dot-dashed line gives the approximate evolution parameter that corresponds to the bifurcation period above which systems evolve towards long orbital periods rather than short periods (see e.g. Pylyser \& Savonije 1989 and PRP). Hence systems between these two lines are potential candidates for AM CVn stars. The value of $f_{\text {rel }}$ in each panel gives the fraction of systems in each mass bin (listed only for the standard case). Note that the number of systems with $M \geq 0.8 \mathrm{M}_{\odot}$ is roughly the same as the number of systems with less massive secondaries, which have dominated in previous CV population synthesis studies.

In Table 2 we present the birthrates in the Galaxy (per year) for the total population of CVs, for CVs that have minimum periods shorter than the standard minimum periods (as indicated in the heading), and CVs with evolved secondaries (with an initial central hydrogen abundance
$\left.X_{c}<0.4\right)$ for our eight main BPS simulations and for two additional simulations where we assumed an age of $15 \mathrm{Gyr}$ and a metallicity of $Z=0.001$ to illustrate the age and metallicity dependence of the results, respectively. In interpreting this table, two things need to be kept in mind: first, it does not include CVs with either He white dwarfs or secondaries with $M<0.35 \mathrm{M}_{\odot}$, since these are not represented in our grid of evolutionary sequences. Second, since all our evolutionary sequences assume a magnetic-braking efficiency of 1 (when the secondaries have a convective envelope and a radiative core) and since the bifurcation period depends on the magnetic-braking law (see Pylyser \& Savonije 1989 and PRP), only models (1), (2) and (1') can be considered fully self-consistent.

Table 2 shows that the birthrate for CVs varies quite substantially for different models from $2 \times 10^{-3} \mathrm{yr}^{-1}$ to $10 \times 10^{-3} \mathrm{yr}^{-1}$ where the value for our standard model falls in the middle $\left(6 \times 10^{-3} \mathrm{yr}^{-1} ;\right.$ model 1$)$. The qualitative behaviour of these birthrates is easy to understand, since less efficient $\mathrm{CE}$ ejection produces systems with shorter orbital periods and more efficient magnetic braking brings them into contact faster. Both effects increase the birthrates of CVs. These Galactic birthrates can be converted into local birthrate densities by dividing them by an effective Galactic volume $5 \times 10^{11} \mathrm{pc}^{3}$; this assumes a local white-dwarf birthrate of $2 \times 10^{-12} \mathrm{pc}^{-3} \mathrm{yr}^{-1}$ (Weidemann 1990), since our star-formation rate produces roughly 1 white dwarf per year in the Galaxy. These numbers therefore 'predict' a local birthrate density of $0.4-2 \times 10^{-14} \mathrm{pc}^{-3} \mathrm{yr}^{-1}$ in good agreement with the observational estimate for the total CV population by Ritter \& Burkert (1986).

Table 2 also shows that the number of CVs with initially evolved secondaries and systems that become ultra-compact represent roughly 10 per cent of the total CV population (see $\S 4$ for further discussion).

\subsection{Simulations of the properties of the CV population}

To simulate the properties of CVs as a function of orbital period, we need to know the relative importance of each of 


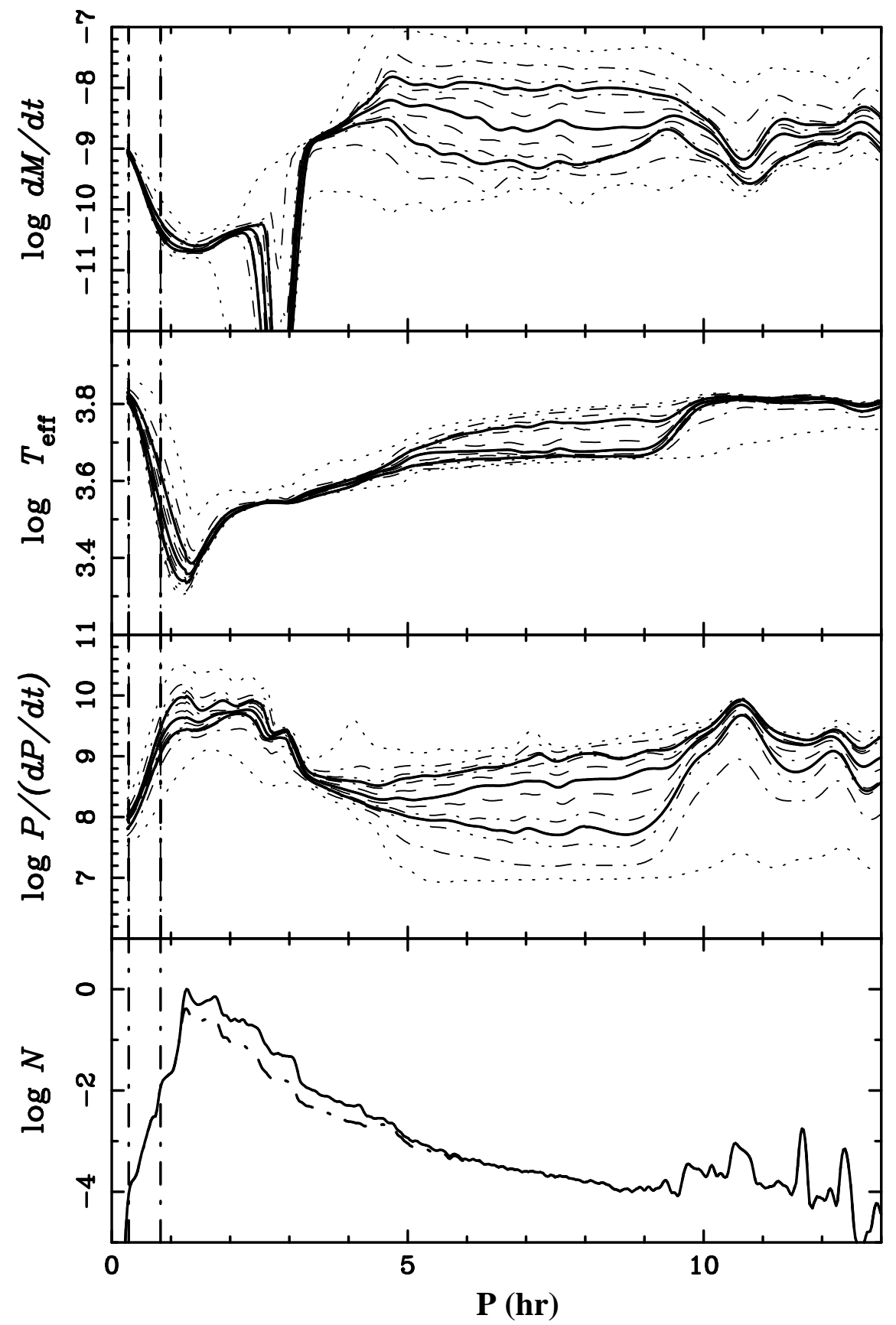

Figure 4. Intrinsic distributions of mass-transfer rate, $\dot{M}\left(\mathrm{M}_{\odot} \mathrm{yr}^{-1}\right)$, effective temperature, $T_{\text {eff }}(\mathrm{K})$, timescale for orbital period change, $P /|\mathrm{d} P / \mathrm{d} t|(\mathrm{yr})$ and total number distribution as a function of orbital period, $P_{\text {orb }}$ for all systems before they have reached their respective minimum period (no selection effects applied). In the top three panels, the thick, solid central curve gives the median of the respective distribution; the pairs of curves moving progressively outwards from the median include 20, 40, 50, 60, 80 and 98 per cent of the distributions at a given orbital period. The regions between the thick solid curves contain 50 per cent of all systems around the median. In the bottom panel the dot-dashed curve indicates the number of systems that initially had evolved secondaries (with $X_{c}<0.4$ ) or secondaries with mass $M_{2} \geq 1 \mathrm{M}_{\odot}$. Note that these systems completely dominate the distribution for orbital periods below $\sim 1.25$ hr and above $\sim 4.7 \mathrm{hr}$. All curves have been smoothed to some degree to reduce the effects of sampling artefacts.

our 120 binary evolution sequences. These sequences span a regular grid of white dwarf and secondary masses $\left(M_{1}\right.$ and $M_{2}$, respectively) as well as evolutionary state of the donor, specified by the parameter $r$ (see eq. 2), when the CV mass-transfer phase commences (see $\S 2$ for details). We determine these relative weights using the BPS results dis- cussed above, which gives us the three-dimensional probability distribution of CVs at the beginning of mass transfer as a function of $M_{1}, M_{2}$, and $r$. For this purpose, we divide this three-dimensional parameter space into a regular three-dimensional grid and determine the relative probability for each of these grid elements. For each system to 
emerge from the BPS code we locate the nearest 8 grid cells ( 2 in each dimension) in our binary evolution sequences and do a tri-linear interpolation to attribute weights to each of these. For systems with white-dwarf masses below $0.6 \mathrm{M}_{\odot}$, we use the sequences with $M_{1}=0.6 \mathrm{M}_{\odot}$ (note that there are very few systems below $0.55 \mathrm{M}_{\odot}$, since we excluded systems with He white dwarfs). For systems with white-dwarf masses above $1.0 \mathrm{M}_{\odot}$, we use the sequences with $1 \mathrm{M}_{\odot}$ (there are also very few such massive systems). For secondaries with initial masses below $0.6 \mathrm{M}_{\odot}$, we use the sequences with $M_{2}=0.6 \mathrm{M}_{\odot}$, but only consider the part of the sequence below the appropriate lower mass.

Having determined the relative weights of the 120 binary evolution sequences, we can then construct the distributions of the CV properties as a function of orbital period directly from these sequences, where we also take into account the time (in yr) a system in each sequence spends at a particular orbital period.

Figure 4 shows the resulting distributions of the masstransfer rate from the secondary $\dot{M}$, its effective temperature, $T_{\text {eff }}$, the timescale for orbital period change, and the total number distribution as a function of orbital period (note that we included only phases before the period minimum for clarity). The shaded regions in the top three panels indicate the range of parameters that include 50 per cent of all systems at a particular orbital period around the median. One of the most striking features of these distributions is that above $\sim 4.7 \mathrm{hr}$ they are extremely wide, e.g. spanning several orders of magnitude in $\dot{M}$. As the shaded area in the bottom panel shows, at these orbital periods, the distributions are completely dominated by systems where the secondary was either evolved initially (with $X_{c}<0.4$ ) or relatively massive (with $M_{2} \geq 1 \mathrm{M}_{\odot}$ ). The large variation of properties is therefore a direct consequence of having a mixture of systems with different initial masses and initial evolutionary states. At a given orbital period, a system with a relatively more massive secondary will generally have a higher mass-transfer rate, be more out of thermal equilibrium and evolve more quickly. On the other hand, for fixed initial masses, the initially more evolved secondary will have a lower mass-transfer rate at a particular orbital period (as can be clearly seen in Fig. 1 by comparing $\dot{M}$ at a particular orbital period; see also Beuermann et al. [1998] and Baraffe \& Kolb [2000] for similar discussions). Since this has the consequence that they evolve more slowly at these periods, they tend to contribute more to the overall distribution (see $\S 4.2$ ). Thus, for example, although only $\sim 15 \%$ of CVs which commence mass transfer with an orbital period $\gtrsim 5 \mathrm{hr}$, have evolved secondaries, the longer dwell times (i.e. slower evolution) makes them by far the most numerous CVs to be found in this period range.

Note that there is a peak in the overall number distribution just above $10 \mathrm{hr}$. This peak is caused by systems that started mass transfer near the bifurcation period. These tend to spend a large fraction of the evolution in this period range (see the dot-dot-dot-dashed and dot-dashed curves in Fig. 1) because of the approximate balancing of opposing effects that drive their period evolution: the angular-momentum losses due to magnetic braking and gravitational radiation that drive the systems towards shorter orbital periods and the effects of mass transfer and nuclear evolution that tend to lead to an increase in the orbital period (once the mass ratio has been reversed).

Below the orbital period of $4.7 \mathrm{hr}$, all the distributions are fairly narrow until they reach the standard period minimum ( $75 \mathrm{~min})$. The main reason for this convergence is that below $4.7 \mathrm{hr}$ the distribution is dominated by initially unevolved systems. As is well known, all evolutionary tracks for normal CVs tend to converge after the initial turn-on phase or initial phase of thermal-timescale mass transfer (see e.g. Rappaport et al. 1983; Stehle, Ritter \& Kolb 1996). Even the systems with initially evolved secondaries tend to converge towards this universal track (see Fig. 1), since at these periods the evolution is mainly driven by angular-momentum loss due to magnetic braking. Since the observed distribution of $\dot{M}$ tends to be much wider, this requires an additional mechanism as, for example, variability induced by nova events which lead to semi-periodic jumps in the location of the Roche lobe in the stellar atmosphere of the secondary (see e.g. Kolb et al. 2001, and references therein). Below the standard period minimum, the distributions widen again because of a large variation of the properties of the secondaries at a given period (e.g. effective temperature, mass, surface abundances). At the shortest orbital periods, $\dot{M}$ starts to increase quite sharply since the timescale for gravitational radiation, which drives the evolution at this stage, decreases rapidly. Note also that the temperature distribution is peaked towards higher temperatures just as the secondaries in individual sequences become hotter (see Fig. 1).

\section{DISCUSSION}

\subsection{The formation of ultra-compact white-dwarf binaries and AM CVn stars}

A substantial fraction of CVs in our simulations (typically 10 per cent of the total sample, excluding CVs with He WDs) evolve towards periods shorter than the classical period minimum. These ultra-compact CVs are excellent candidates for AM CVn stars. To assess their relative importance, we need to compare this channel to the alternative channels discussed in the past, the double-degenerate channel and the helium-star channel (see $\S 1$ ). NPVY have done the most thorough BPS analysis of these channels to-date, including a detailed assessment of the uncertainties in the theoretical modelling. Their estimated Galactic birthrates vary from $0.04-4.7 \times 10^{-3} \mathrm{yr}^{-1}$ for the double-degenerate model and from $0.9-1.6 \times 10^{-3} \mathrm{yr}^{-1}$ for the helium-star model (see table 1 in NPVY). In comparison, we obtain birthrates from $0.5-1.3 \times 10^{-3} \mathrm{yr}^{-1}$ (see Table 2 ). These numbers are, however, not directly comparable. First, while NPVY assume a similar star-formation rate (by mass) at the present epoch (3.6 versus $3.5 \mathrm{M}_{\odot} \mathrm{yr}^{-1}$ ), their star-formation rate was much higher in the past, starting with a star-formation rate of $15 \mathrm{M}_{\odot} \mathrm{yr}^{-1}$ and decaying with an exponential decay time of 7 Gyr (Nelemans et al. 2001b). For their adopted Galactic age of $10 \mathrm{Gyr}$, this gives an average star-formation rate of $8 \mathrm{M}_{\odot} \mathrm{yr}^{-1}$. Since in our simulations, the sample of AM CVn stars is dominated by relatively old systems, this would suggest that one should multiply our numbers by a factor of 2 to 4 for the purpose of comparing the two models (there are 
several other differences in the model assumptions which cumulatively make only a minor difference of order 1 ). On the other hand, only a fraction of our systems (roughly 20 per cent), reach very short periods ( $\lesssim 45 \mathrm{~min}$ ), while all systems in the study of NPVY are guaranteed to do so. Considering all the uncertainties in these various estimates, we think that a fair conclusion is that the $\mathrm{CV}$ channel provides a potentially important channel for the formation of AM CVn stars, competitive with the double-degenerate and the helium-star channel, although it may or may not be the dominant one.

In Table 1 we list a number of model parameters for the six well-established AM CVn stars, for systems before and after their period minimum, respectively. The quoted ranges for these parameters include 90 per cent of all systems around the median at the respective orbital period, although we note that, at the shortest orbital periods, these are almost certainly underestimates since this period range is not very well sampled in our simulations. In the last two columns we also list the mass estimates for these 6 systems from both the double-degenerate (DD) and the helium-star model (He), again taken directly from NPVY. The differences in masses can be largely attributed to differences in composition, the degree of degeneracy and possibly some differences in the assumed equation of state (implicit in the approximate mass - radius relation for degenerate stars in NPVY). As already noted earlier, one possible way of distinguishing between the $\mathrm{CV}$ channel and the alternative channels for $\mathrm{AM} \mathrm{CVn}$ stars is that, in the CV channel, AM CVn stars still have some hydrogen left in their envelopes when they approach the period minimum, but very little or none after the period minimum (see column ' $X$ ' in Table 1). As far as we are aware, of the $6 \mathrm{AM} \mathrm{CVn} \mathrm{stars} \mathrm{in} \mathrm{the} \mathrm{table,} \mathrm{only} \mathrm{one,} \mathrm{GP}$ Com, has a sufficiently good spectrum that it is confirmed to be very hydrogen-deficient; Marsh, Horne \& Rosen (1991) have determined an upper limit on the ratio of hydrogen to helium abundance (by number) of $\mathrm{H} / \mathrm{He}<10^{-5}$.

Since we are likely to see only a small fraction of the total population of AM CVn stars, a detailed comparison of the simulations with observations is seriously compromised by severe selection effects (as is the population of ordinary CVs; see e.g. HNR). NPVY have attempted to model these in a reasonably physical manner. Here, for the purpose of illustration, we adopt a more ad hoc procedure (also see HNR for discussion). To simulate observational number distributions, we multipy the actual number of systems by an observing efficiency, $f_{\text {obs }}$, which mainly depends on the mass-transfer rate $\dot{M}$, i.e., $f_{\text {obs }} \propto \dot{M}^{\gamma}$ (for $\left.\dot{M} \leq 4 \times 10^{-10} \mathrm{M}_{\odot} \mathrm{yr}^{-1}\right)$, where the power in the exponent increases from 1 (below $\dot{M} \leq 4 \times 10^{-10} \mathrm{M}_{\odot} \mathrm{yr}^{-1}$ ) to 2 (below $\dot{M} \leq 1 \times 10^{-10} \mathrm{M}_{\odot} \mathrm{yr}^{-1}$ ), and finally 3 (below $\left.\dot{M} \leq 4 \times 10^{-\overline{11}} \mathrm{M}_{\odot} \mathrm{yr}^{-1}\right)$. We also assume that very short orbital periods are easier to determine and that the detection efficiency, below an orbital period of $2 \mathrm{hr}$, increases $\propto P_{\text {orb }}^{-1}$. We emphasize that this observational selection criterion is not based on a quantitative physical model, but was constructed mainly to get (1) a number distribution that has a similar appearance to the observed one (Ritter \& Kolb 1998), (2) reduces the spike near the standard period minimum, and (3) shows the behaviour at ultra-short periods clearly. The main purpose of this exercise is to illustrate the dependence of the number of observable systems on the various model parameters. Note also that this selection criterion

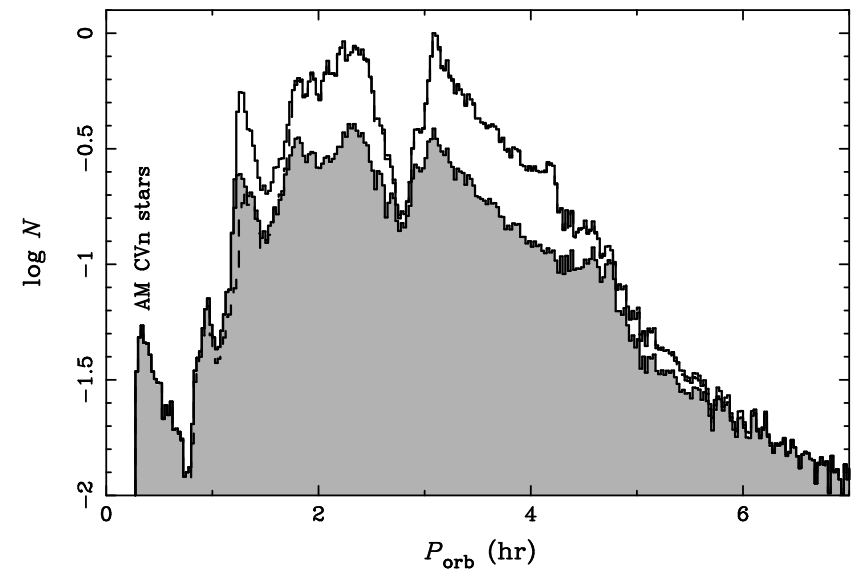

Figure 5. Simulated number distribution for systems as a function of orbital period with our ad hoc observational selection criterion for model (1). The lightly shaded area shows the distribution of systems that started either with an evolved secondary (with $X_{c}<0.4$ ) or a secondary of mass $\geq 1^{*} \mathrm{M}_{\odot}$. The dashed histogram, only distinct around an orbital period of $1.25 \mathrm{hr}$, shows the distribution where systems with $\dot{M} \leq 2.5 \times 10^{-11} \mathrm{M}_{\odot} \mathrm{yr}^{-1}$ were eliminated from the observable sample. Note that systems with $P_{\text {orb }} \gtrsim 4.7 \mathrm{hr}$ and $P_{\text {orb }} \lesssim 1.25 \mathrm{hr}$ are largely ones that started with evolved or more massive secondaries. The region of the simulated AM CVn systems is indicated on the figure.

does not strongly affect systems above the period gap, which mostly have much higher values for $\dot{M}$ (see Fig. 4)).

Figure 5 shows the simulated number distribution with this selection criterion for model (1) where the lightly shaded region indicates the distribution of systems that started either with an initially evolved secondary (with $X_{c}<0.4$ ) or a secondary of mass $\geq 1 \mathrm{M}_{\odot}$. As already discussed in $\S 3.3$, these evolved/massive systems completely dominate the distribution below $\sim 1.25 \mathrm{hr}$ and above $\sim 4.7 \mathrm{hr}$. While they also fill in part of the period gap (between $\sim 2.5$ and $\sim 3 \mathrm{hr}$ in our evolution model; see $\S 2$ ), the period gap still remains well defined. (Note, however, that CVs with He white dwarfs and CVs that start mass transfer in the orbital period range will further increase the number of systems in the period gap; see e.g. HNR). Around an orbital period of $75 \mathrm{~min}$, the distribution shows the well-known period-minimum spike (since the orbital period evolves slowly near the period minimum). This spike is not apparent in the observed distribution, perhaps suggesting more drastic selection effects (see the detailed discussion of this problem in Kolb \& Baraffe 1999). To illustrate this possibility, we performed another simulation where we assumed that no systems with $\dot{M}$ below $2.5 \times 10^{-10} \mathrm{M}_{\odot} \mathrm{yr}^{-1}$ were detectable. This simulation is shown as a dashed histogram in Figure 5 and is only clearly visible near the period minimum. The only significant change this additional assumption introduces is that it more or less completely eliminates the period-minimum spike. However, this requires significant fine-tuning because the range of mass-transfer rates near the period minimum is very small, and therefore this cannot be considered very plausible. On the other hand, if there were a larger range of $\dot{M}$ near the period minimum or a larger variation of the minimum period (which is not present in current evolutionary models), one can imagine that the spike would be spread out. A more drastic alternative, suggested by King (2001), 
Table 3. Predicted number distributions for CVs with CO white dwarfs with our ad hoc selection criterion (normalized to the total of $\sim 400$ currently known systems).

\begin{tabular}{ccccccccccc}
\hline \hline & & \multicolumn{10}{c}{$P(\mathrm{hr})$} & & \\
\cline { 4 - 9 }$f_{\mathrm{MB}}$ & $\alpha_{\mathrm{CE}}$ & $\alpha_{\text {th }}$ & $0-0.42$ & $0.42-0.75$ & $0.75-1.17$ & $1.17-2.5$ & $2.5-3.0$ & $3.0-5.0$ & $>5.0$ & model \\
\hline \multirow{2}{*}{1} & 1 & 1 & 1.7 & 1.9 & 5.1 & 173 & 37 & 156 & 25 & $(1)$ \\
& 0.5 & 0 & 1.1 & 1.5 & 6.5 & 176 & 36 & 160 & 19 & $(2)$ \\
0 & 1 & 1 & 7.6 & 6.3 & 10.4 & 162 & 40 & 139 & 34 & $(3)$ \\
& 0.5 & 0 & 8.5 & 6.8 & 11.3 & 163 & 37 & 142 & 33 & $(4)$ \\
0.2 & 1 & 1 & 2.2 & 2.4 & 6.2 & 170 & 39 & 153 & 27 & $(5)$ \\
& 0.5 & 0 & 2.3 & 2.3 & 7.5 & 174 & 37 & 156 & 21 & $(6)$ \\
5 & 1 & 1 & 1.4 & 1.5 & 4.2 & 175 & 35 & 159 & 24 & $(7)$ \\
& 0.5 & 0 & 0.0 & 0.6 & 4.6 & 180 & 36 & 164 & 15 & $(8)$ \\
1 & 1 & 1 & 1.7 & 1.8 & 5.1 & 173 & 37 & 156 & 25 & $(1)$ \\
1 & 1 & 1 & 1.4 & 1.8 & 4.7 & 165 & 44 & 149 & 34 & $\left(1^{*}\right)$ \\
\hline
\end{tabular}

would be that CVs never reach the minimum period in the lifetime of the Galaxy, in which case the observed cut-off near $80 \mathrm{~min}$ is an age effect. However, this suggestion is not consistent with standard BPS assumptions as employed in the present and other related studies and would require some drastic alterations to the entire BPS model.

Below the minimum period, there is a further spike in the orbital-period distribution around $20 \mathrm{~min}$, the period range where most AM CVn stars are observed. Even though the number of actual systems decreases monotonically with decreasing orbital period, the mass-transfer rates go up sharply, which makes the systems with ultra-short periods easier to detect observationally. This 'AM CVn' spike is therefore very sensitive to the observational selection criterion (it is also present in the study of NPVY who use a more physical model for the observational detectibility of their systems).

In Table 3 we present the number distribution in various period ranges for all of the BPS models using our ad hoc selection criterion, normalized to a total number of 400 systems (approximately the number of known CVs). This illustrates the variation in the number of observable systems with the BPS parameters. It shows that the number of observable AM CVn stars (resulting only from the CV channel) is largest if there is no magnetic braking before the onset of mass transfer and decreases as the efficiency of magnetic braking is increased (see the discussion in $\S 3.2$ ). There is generally a good correlation between the number of systems with periods above $5 \mathrm{hr}$ (dominated by evolved/massive systems), systems in the period gap, and ultra-compact systems.

Finally we note that the number distributions are also somewhat sensitive to other factors which we did not vary systematically, in particular the assumed age of the Galaxy and the metallicity of the secondaries. Since AM CVn stars in our model originate from systems where the secondaries start mass transfer near the end of, or just after, their mainsequence phase, the age of the Galaxy determines the lowest initial mass of a secondary that can evolve to the AM CVn stage. Similarly, since low-metallicity stars evolve much more rapidly, population-II stars of lower mass will be able to evolve to become ultra-compact systems. In Tables 2 and 3 , the models $\left(1^{\prime}\right)$ and $\left(1^{*}\right)$ give the birthrates and number distributions for models with a Galactic age of $15 \mathrm{Gyr}$ and for a population II metallicity with $Z=0.001$ (for an age of $12 \mathrm{Gyr}$ ), where all other parameters were the same as in model (1). simulations.

\subsection{Cataclysmic variables with evolved companions}

In Figure 6 we present a comparison of our simulated distribution of the effective temperature of the secondary as a function of orbital period with the spectral types of secondaries from the sample of Beuermann et al. (1998). To plot the observed systems, we used the spectral type - effective temperature relation of Schmidt-Kaler (1982) and added (in quadrature) an error of $300 \mathrm{~K}$, due to the uncertainties in this relation at late spectral types, to the errors in the spectral-type determination given by Beuermann et al. (1998). In addition, since our stellar models tend to overestimate the effective temperature below $\sim 4500 \mathrm{~K}$ compared to evolutionary calculations with a more sophisticated treatment of the stellar atmosphere (see Chabrier \& Baraffe 1997; Baraffe et al. 1998), we applied corrections to our effective temperatures to make the comparison more realistic. These corrections were obtained by comparing the effective temperatures of unevolved stars between 0.1 and $1 \mathrm{M}_{\odot}$ in our models with the models of Baraffe et al. (1998). The difference between the dashed and the dot-dashed curves in Figure 6 shows the magnitude of this correction for unevolved stars in thermal equilibrium, which start to fill their Roche lobes at a particular orbital period (the correction has a maximum of $350 \mathrm{~K}$ around $4000 \mathrm{~K})^{3}$.

As Figure 6 shows, our simulations reproduce the observed distribution reasonably well at all orbital periods. In particular, they show a large variation of effective temperatures/spectral types at orbital periods longer than $\sim 5 \mathrm{hr}$, consistent with the observed variation and confirming the suggestion by Beuermann et al. (1998) and Baraffe \& Kolb (2000) that systems at these periods are dominated by those with evolved secondaries. Many of these systems may therefore show evidence for nuclear processing (in particular CNO

3 We note that the determination of this correction is not entirely straightforward, since the Baraffe et al. (1998) models have not been calibrated to reproduce a solar model in a self-consistent way. 


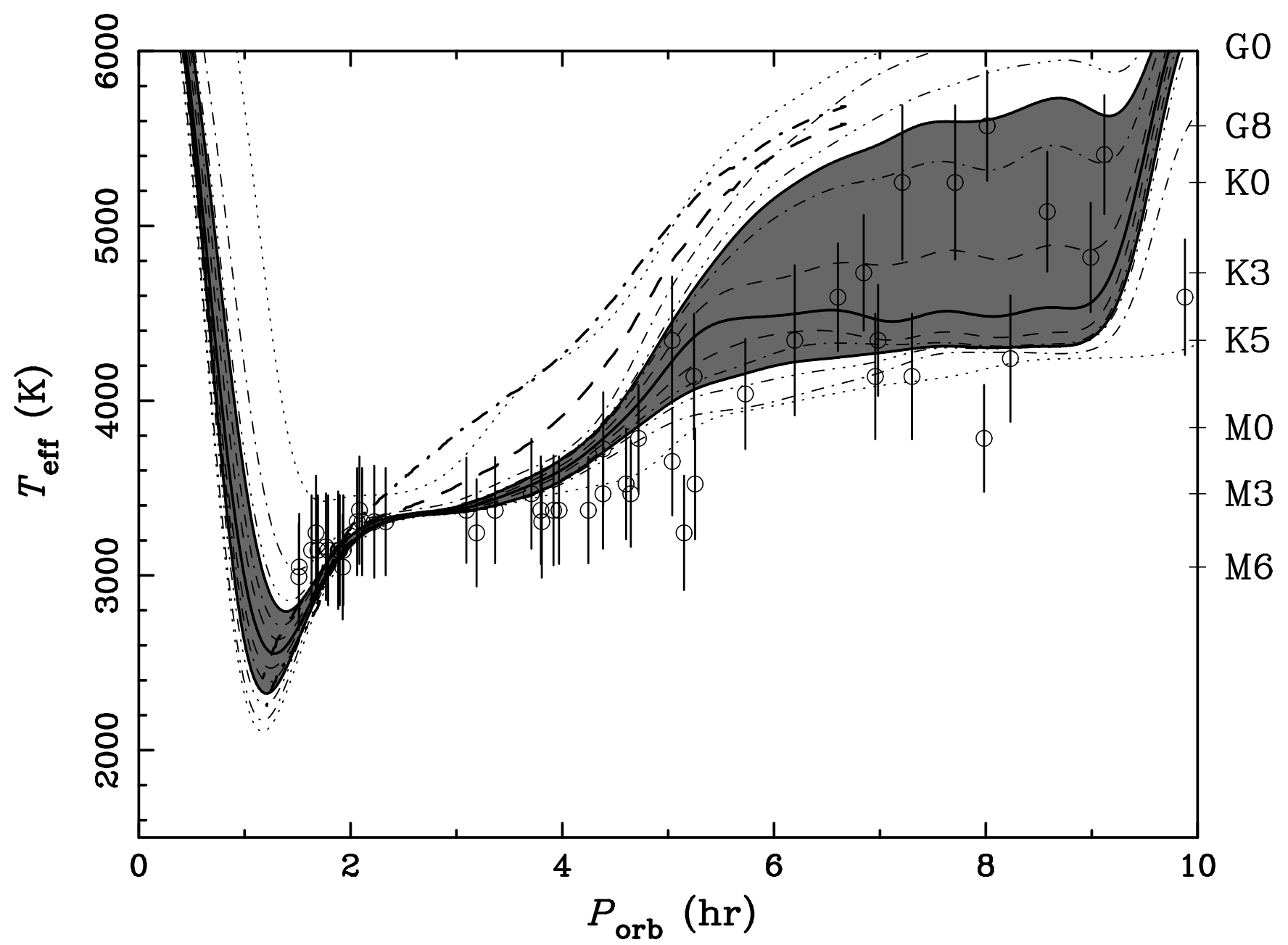

Figure 6. Comparison of the theoretical distribution of effective temperatures/spectral type as a function of orbital period with the observational data of Beuermann et al. (1998). The various curves show the intrinsic distribution of temperatures for model (1) (as in Fig. 4), where the shaded region includes 50 per cent of all systems around the median (only for systems before they reached the period minimum). The circles with error bars give the temperatures of systems with spectral types taken from Beuermann et al. (1998). The thick dashed curve shows the temperature of systems that are unevolved and in thermal equilibrium if they fill their Roche lobes at a particular orbital period (assuming a white dwarf of $0.7 \mathrm{Msun}$ and with temperature corrections applied). The dot-dashed curve shows the temperature of the same systems without temperature corrections applied (see text for details).

processing) in their envelopes. Below an orbital period of $\sim 2 \mathrm{hr}$, the observed secondaries are close to the relation for unevolved stars in thermal equilibrium (the thick dashed curve) as expected in the standard model for CVs, where the secondaries remain very close to thermal equilibrium until near the end of their hydrogen-burning phase. Near an orbital period of $\sim 5 \mathrm{hr}$, the observed spectral types appear to be slightly, but systematically later than our simulations imply. The reason may be that the applied effective temperature corrections were not large enough or that our stars are not sufficiently out of thermal equilibrium in this period range (perhaps consistent with having a period gap that is slightly too small; see $\S 2$; for a different interpretation see Baraffe \& Kolb 2000). We also note that for orbital periods longer than $\sim 5 \mathrm{hr}$, some of the observed systems could originate from more massive, supersoft systems (not included in our simulations), which under certain conditions may reach orbital periods as short as $4 \mathrm{hr}$ (King et al. 2001) before the period increases again. However, since this occurs during a fast evolutionary phase with large $\dot{M}$, they are unlikely to dominate the distribution below orbital periods of $\sim 10 \mathrm{hr}$, which in our simulations are dominated by systems with relatively low $\dot{M}$ with evolved secondaries (see Figures 1 and 4).

\section{CONCLUSION}

We have presented a self-consistent study of CVs and CVlike systems, combining detailed binary population synthesis simulations with a large grid of binary evolutionary calculations, where we included systems with evolved secondaries up to a mass of $1.4 \mathrm{M}_{\odot}$. These simulations show that CVs above $\sim 5 \mathrm{hr}$ are dominated by evolved, relatively massive systems and that the predicted range in effective temperatures is consistent with the variation in spectral types obtained by Beuermann et al. (1998). These systems, which constitute $\sim 10$ per cent of the total CV population, are also the progenitors of ultra-compact systems with periods 
less than $\sim 1 \mathrm{hr}$ which are good candidates for $\mathrm{AM} \mathrm{CVn}$ stars. For standard population synthesis assumptions, we obtain AM CVn birthrates that are competitive with those in alternative models. While these systems do not experience a period gap between 2 and $3 \mathrm{hr}$, their contribution is sufficiently small that they do not destroy its distinct appearance.

\section{ACKNOWLEDGEMENTS}

This work was in part supported by a Royal Society UKChina Joint Project Grant (Ph.P and Z.H.), the Chinese National Science Foundation under Grant No. 19925312, 10073009 and NKBRSF No. 19990754 (Z.H.) and by the National Aeronautics and Space Administration under ATP grant NAG5-8368.

\section{REFERENCES}

Baraffe I., Chabrier G., Allard F., Hauschildt P.H., 1998, A\&A, 337, 403

Baraffe I., Kolb U., 2000, MNRAS, 318, 354

Beuermann K., Baraffe I., Kolb U., Weichold M., 1998, A\&A, 339,518

Chabrier G., Baraffe I., 1997, A\&A, 327, 1039

Cropper M., Harrop-Allin M.K., Mason K.O., Mittaz J.P.D., Potter S.B., Ramsay G., 1998, MNRAS, 293, L57

de Kool M., 1992, A\&A, 261, 188

Dewi J., Tauris T., 2000, A\&A, 360, 1043

Evans C.R., Iben I.Jr., Smarr L., 1987, ApJ, 323, 129

Faulkner J., Flannery B.P., Warner B., 1972, ApJ, 175, L79

Fedorova A.V., Ergma E.V., 1989, Ap\&SS, 151, 125

Han Z., 1995, Ph.D. Thesis (Cambridge), unpublished

Han Z., 1998, MNRAS, 296, 1019

Han Z., Eggleton P.P., Podsiadlowski Ph., Tout C.A., 1995, 277, 1443

Han Z., Eggleton P.P., Podsiadlowski Ph., Tout C.A., Webbink R.F., 2001, in Podsiadlowski Ph., Rappaport S., King A.R., D'Antona F., Burderi L., eds, Evolution of Binary and Multiple Star Systems, ASP Conf. Ser., Vol. 229, p. 205

Han Z., Podsiadlowski Ph., Eggleton P.P., 1994, MNRAS, 270, 121

Han Z., Podsiadlowski Ph., Eggleton P.P., 1995, MNRAS, 272, 800 (HPE)

Han Z., Webbink R.F., 1999, A\&A, 349, L17

Hils D., Bender P.L., Webbink R.F., 1990, ApJ, 360, 75; 369, 271

Howell S.B., Nelson, L.A., Rappaport S., 2001, ApJ, 550, 897 (HNR)

Iben I.Jr., Tutukov A.V., 1984, ApJS, 54, 335

Iben J.Jr., Tutukov A.V., 1991, ApJ, 370, 615

King A.R., 2001, talk presented at meeting "The Physics of Cataclysmic Variables and Related Objects", Göttingen, August 2001

King A.R., Schenker K., Kolb U., Davies M.B., 2001, MNRAS, 321,327

Kippenhahn R., Weigert A., Hofmeister E., 1967 in Alder B., Fernbach S., Rothenberg M., Methods in Computational Physics, Vol. 7, p. 129

Kolb U., 1993, A\&A, 271, 149

Kolb U., Baraffe I., 1999, MNRAS, 309, 1034

Kolb U., Rappaport S.. Schenker K., Howell S., 2001, ApJ, in press astro-ph/0108322

Langer N., Deutschmann A., Wellstein S., Höflich P., 2000, A\&A, 361,1046
Marsh T.R., Horne K., Rosen S., 1991, ApJ, 366, 535

Miller G.E., Scalo J.M., 1979, ApJS, 41, 513

Nather R.E., Robinson E.L., Stover R.J., 1981, ApJ, 244, 269

Nelemans G., Portegies Zwart S.F., Verbunt F., Yungelson L.R., 2001a, A\&A, 368, 939 (NPVY)

Nelemans G., Yungelson L.R., Portegies Zwart S.F., Verbunt F., 2001b, A\&A, 365, 491

Paczyński B., 1967, Acta Astron., 17, 287

Paczyński B., 1976, in Eggleton P.P., Mitton S., Whelan J., eds, Structure and Evolution of Close Binaries. Kluwer, Dordrecht, p. 75

PodsiadlowskiPh. Rappaport S., Pfahl E., 2001, ApJ, submitted (astro-ph/0107261) (PRP)

Politano M., 1996, ApJ, 465, 338

Pylyser E.H.P., Savonije G.J., 1989, A\&A 208, 52

Rappaport S., Di Stefano R., Smith M., 1994, ApJ, 426, 692

Rappaport S., Verbunt F., Joss P.C., 1983, ApJ, 275, 713

Ritter H., Burkert A., 1986, A\&A, 158, 161

Ritter H., Kolb U., 1998, A\&AS 129, 83

Savonije G.J., de Kool M., van den Heuvel E.P.J., 1986, A\&A, 155,51

Schmidt-Kaler Th., 1982, in Scaifers K., Voigt H.H., eds, Numerical Data and Functional Relationship in Science and Technology, Vol. 2(b), Springer, Berlin, p. 453

Shafter A.W., 1992, ApJ, 394, 268

Smak J., 1967, Acta Astron., 17, 255

Smith, D.A., Dhillon V.S., 1998, MNRAS, 301, 767

Spruit H.C., Ritter H., 1983, A\&A, 124, 267

Stehle R., Ritter H., Kolb U., 1996, MNRAS, 279, 518

Tutukov A.V., Fedorova A.V., Ergma E., Yungelson L., 1987, Sov. Astron. Lett., 13, 328

Tutukov A.V., Yungelson L.R., 1979, Acta Astron., 29, 665

Verbunt F., Zwaan C., 1981, A\&A, 100, L7

Warner B., 1995, Ap\&SS, 225, 249

Warner B., Robinson E.L., 1972, MNRAS, 159, 101

Weidemann V., 1990, ARA\&A, 28, 103

Webbink R.F., 1984, ApJ, 277, 355

\section{APPENDIX A: APPENDIX}

Table A1 lists some of the parameters which are important in the present study for the 120 sequences in our grid. These include the initial parameters, parameters at the period minimum and the size of the period gap, if present. (For the definitions of the parameters, see the bottom of the table.) The last column gives the relative weight of each sequence in the CV population synthesis for BPS model (1). 


\section{Podsiadlowski, Han \& Rappaport}

Table A1. Selected Properties of Binary Sequences

\begin{tabular}{|c|c|c|c|c|c|c|c|c|c|c|c|}
\hline \multicolumn{6}{|c|}{ Initial Parameters } & \multicolumn{4}{|c|}{ Parameters at Period Minimum } & \multirow[b]{2}{*}{$\begin{array}{l}\text { gap } \\
(\mathrm{hr})\end{array}$} & \multirow[b]{2}{*}{ weight } \\
\hline $\begin{array}{c}M_{1} \\
\left(\mathrm{M}_{\odot}\right)\end{array}$ & $\begin{array}{c}M_{2} \\
\left(\mathrm{M}_{\odot}\right)\end{array}$ & $\begin{array}{c}P^{i} \\
(\mathrm{hr})\end{array}$ & $\begin{array}{c}t^{i} \\
(\mathrm{yr})\end{array}$ & $X_{c}^{i}$ & $M_{c}^{i} / M$ & $\begin{array}{l}P_{\min } \\
(\min )\end{array}$ & $\begin{array}{l}M_{2}^{\min } \\
\left(\mathrm{M}_{\odot}\right)\end{array}$ & $\begin{array}{c}\dot{M}_{\min } \\
\left(\mathrm{M}_{\odot} \mathrm{yr}^{-1}\right)\end{array}$ & $X_{s}^{\min }$ & & \\
\hline 0.6 & 0.6 & 4.1 & $0.0 \mathrm{E}+00$ & 0.685 & 0.000 & 74.8 & 0.069 & $2.1 \mathrm{E}-11$ & 0.682 & $2.52-3.08$ & $1.0 \mathrm{E}-01$ \\
\hline 0.6 & 0.8 & 5.3 & $0.0 \mathrm{E}+00$ & 0.685 & 0.000 & 75.2 & 0.067 & $2.0 \mathrm{E}-11$ & 0.682 & $2.46-3.07$ & $5.0 \mathrm{E}-02$ \\
\hline 0.6 & 0.8 & 5.5 & $3.9 \mathrm{E}+09$ & 0.560 & 0.000 & 74.1 & 0.063 & $1.9 \mathrm{E}-11$ & 0.662 & $2.33-2.92$ & $4.6 \mathrm{E}-02$ \\
\hline 0.6 & 0.8 & 5.7 & $8.0 \mathrm{E}+09$ & 0.436 & 0.000 & 73.3 & 0.056 & $1.6 \mathrm{E}-11$ & 0.638 & $1.75-2.07$ & $9.5 \mathrm{E}-03$ \\
\hline 0.6 & 0.9 & 5.8 & $0.0 \mathrm{E}+00$ & 0.685 & 0.000 & 75.2 & 0.066 & $2.1 \mathrm{E}-11$ & 0.682 & $2.43-3.07$ & $3.6 \mathrm{E}-02$ \\
\hline 0.6 & 0.9 & 6.1 & $2.7 \mathrm{E}+09$ & 0.552 & 0.000 & 73.8 & 0.066 & $2.1 \mathrm{E}-11$ & 0.659 & $2.31-2.87$ & $2.5 \mathrm{E}-02$ \\
\hline 0.6 & 0.9 & 6.4 & $5.1 \mathrm{E}+09$ & 0.433 & 0.000 & 72.9 & 0.068 & $2.1 \mathrm{E}-11$ & 0.635 & $1.89-2.25$ & $6.7 \mathrm{E}-03$ \\
\hline 0.6 & 0.9 & 6.9 & $7.7 \mathrm{E}+09$ & 0.310 & 0.000 & 70.6 & 0.055 & $1.9 \mathrm{E}-11$ & 0.594 & $1.35-1.72$ & $5.5 \mathrm{E}-03$ \\
\hline 0.6 & 0.9 & 8.1 & $1.2 \mathrm{E}+10$ & 0.093 & 0.000 & 64.7 & 0.045 & $1.6 \mathrm{E}-11$ & 0.491 & $1.22-1.45$ & $2.1 \mathrm{E}-03$ \\
\hline 0.6 & 1.0 & 6.5 & $0.0 \mathrm{E}+00$ & 0.685 & 0.000 & 75.3 & 0.067 & $2.1 \mathrm{E}-11$ & 0.682 & $2.45-3.07$ & $3.0 \mathrm{E}-02$ \\
\hline 0.6 & 1.0 & 6.9 & $1.5 \mathrm{E}+09$ & 0.567 & 0.000 & 73.8 & 0.066 & $2.1 \mathrm{E}-11$ & 0.660 & $2.24-2.90$ & $2.6 \mathrm{E}-02$ \\
\hline 0.6 & 1.0 & 7.3 & $3.2 \mathrm{E}+09$ & 0.444 & 0.000 & 72.8 & 0.060 & $1.9 \mathrm{E}-11$ & 0.635 & $1.93-2.35$ & $7.3 \mathrm{E}-03$ \\
\hline 0.6 & 1.0 & 7.8 & $4.6 \mathrm{E}+09$ & 0.327 & 0.000 & 62.7 & 0.046 & $1.3 \mathrm{E}-11$ & 0.574 & $1.31-1.44$ & $6.1 \mathrm{E}-03$ \\
\hline 0.6 & 1.0 & 8.9 & $7.1 \mathrm{E}+09$ & 0.101 & 0.000 & 54.8 & 0.041 & $1.5 \mathrm{E}-11$ & 0.405 & $1.30-1.45$ & $5.2 \mathrm{E}-03$ \\
\hline 0.6 & 1.0 & 10.8 & $9.0 \mathrm{E}+09$ & 0.000 & 0.000 & 44.6 & 0.031 & $1.4 \mathrm{E}-11$ & 0.196 & & $3.4 \mathrm{E}-03$ \\
\hline 0.6 & 1.0 & 13.2 & $1.0 \mathrm{E}+10$ & 0.000 & 0.037 & 22.1 & 0.065 & $5.8 \mathrm{E}-10$ & 0.053 & & $1.8 \mathrm{E}-03$ \\
\hline 0.6 & 1.0 & 15.4 & $1.1 \mathrm{E}+10$ & 0.000 & 0.063 & 11.1 & 0.100 & $4.7 \mathrm{E}-09$ & 0.037 & & $9.9 \mathrm{E}-04$ \\
\hline 0.6 & 1.0 & 18.2 & $1.1 \mathrm{E}+10$ & 0.000 & 0.093 & & & & & & \\
\hline 0.6 & 1.1 & 7.4 & $0.0 \mathrm{E}+00$ & 0.685 & 0.000 & 75.2 & 0.067 & $2.0 \mathrm{E}-11$ & 0.682 & $2.44-3.08$ & $2.8 \mathrm{E}-02$ \\
\hline 0.6 & 1.1 & 7.9 & $1.1 \mathrm{E}+09$ & 0.558 & 0.000 & 74.0 & 0.062 & $1.9 \mathrm{E}-11$ & 0.657 & $2.32-2.90$ & $2.7 \mathrm{E}-02$ \\
\hline 0.6 & 1.1 & 8.3 & $2.1 \mathrm{E}+09$ & 0.448 & 0.000 & 73.4 & 0.063 & $2.2 \mathrm{E}-11$ & 0.634 & $1.99-2.40$ & $6.4 \mathrm{E}-03$ \\
\hline 0.6 & 1.1 & 8.8 & $2.9 \mathrm{E}+09$ & 0.340 & 0.000 & 71.0 & 0.060 & $2.0 \mathrm{E}-11$ & 0.604 & $1.41-1.79$ & $5.9 \mathrm{E}-03$ \\
\hline 0.6 & 1.1 & 9.8 & $4.3 \mathrm{E}+09$ & 0.105 & 0.000 & 67.3 & 0.048 & $1.5 \mathrm{E}-11$ & 0.537 & $1.38-1.67$ & $5.7 \mathrm{E}-03$ \\
\hline 0.6 & 1.1 & 11.6 & $5.6 \mathrm{E}+09$ & 0.000 & 0.000 & 53.2 & 0.033 & $1.2 \mathrm{E}-11$ & 0.265 & & $5.1 \mathrm{E}-03$ \\
\hline 0.6 & 1.1 & 15.4 & $7.1 \mathrm{E}+09$ & 0.000 & 0.039 & 17.8 & 0.076 & $1.4 \mathrm{E}-09$ & 0.030 & & $3.3 \mathrm{E}-03$ \\
\hline 0.6 & 1.1 & 18.2 & $7.5 \mathrm{E}+09$ & 0.000 & 0.070 & & & & & & \\
\hline 0.6 & 1.2 & 8.4 & $0.0 \mathrm{E}+00$ & 0.685 & 0.000 & 75.8 & 0.071 & $2.4 \mathrm{E}-11$ & 0.682 & $2.45-3.07$ & $2.1 \mathrm{E}-02$ \\
\hline 0.6 & 1.2 & 9.4 & $1.4 \mathrm{E}+09$ & 0.567 & 0.000 & 71.4 & 0.071 & $2.1 \mathrm{E}-11$ & 0.632 & $1.83-2.26$ & $5.7 \mathrm{E}-02$ \\
\hline 0.6 & 1.2 & 10.6 & $2.7 \mathrm{E}+09$ & 0.448 & 0.000 & 66.2 & 0.046 & $1.6 \mathrm{E}-11$ & 0.518 & $1.73-2.05$ & $1.7 \mathrm{E}-02$ \\
\hline 0.6 & 1.2 & 12.0 & $3.7 \mathrm{E}+09$ & 0.329 & 0.000 & 67.3 & 0.047 & $1.5 \mathrm{E}-11$ & 0.523 & $1.79-2.05$ & $1.9 \mathrm{E}-02$ \\
\hline 0.6 & 1.2 & 14.9 & $5.2 \mathrm{E}+09$ & 0.105 & 0.000 & 50.5 & 0.034 & $1.6 \mathrm{E}-11$ & 0.218 & $1.33-1.35$ & $5.9 \mathrm{E}-03$ \\
\hline 0.6 & 1.2 & 15.6 & $5.4 \mathrm{E}+09$ & 0.047 & 0.000 & 34.7 & 0.042 & $6.4 \mathrm{E}-11$ & 0.078 & & $1.6 \mathrm{E}-03$ \\
\hline 0.6 & 1.2 & 16.4 & $5.6 \mathrm{E}+09$ & 0.010 & 0.000 & & & & & & \\
\hline 0.6 & 1.4 & 10.1 & $1.6 \mathrm{E}+07$ & 0.681 & 0.000 & 75.7 & 0.063 & $1.9 \mathrm{E}-11$ & 0.679 & $2.41-3.07$ & $9.2 \mathrm{E}-03$ \\
\hline 0.6 & 1.4 & 11.7 & $9.5 \mathrm{E}+08$ & 0.559 & 0.000 & 72.3 & 0.070 & $2.4 \mathrm{E}-11$ & 0.613 & $2.32-2.86$ & $3.3 \mathrm{E}-02$ \\
\hline 0.6 & 1.4 & 13.4 & $1.7 \mathrm{E}+09$ & 0.448 & 0.000 & 68.9 & 0.048 & $1.4 \mathrm{E}-11$ & 0.544 & $2.20-2.63$ & $1.1 \mathrm{E}-02$ \\
\hline 0.6 & 1.4 & 15.5 & $2.2 \mathrm{E}+09$ & 0.340 & 0.000 & 55.8 & 0.043 & $2.0 \mathrm{E}-11$ & 0.378 & $2.01-2.16$ & $6.2 \mathrm{E}-03$ \\
\hline 0.6 & 1.4 & 18.1 & $2.7 \mathrm{E}+09$ & 0.221 & 0.000 & 47.4 & 0.035 & $2.2 \mathrm{E}-11$ & 0.249 & $1.16-1.25$ & $4.0 \mathrm{E}-03$ \\
\hline 0.6 & 1.4 & 20.3 & $3.1 \mathrm{E}+09$ & 0.112 & 0.000 & 40.5 & 0.046 & $3.5 \mathrm{E}-11$ & 0.109 & & $2.6 \mathrm{E}-03$ \\
\hline 0.6 & 1.4 & 22.3 & $3.3 \mathrm{E}+09$ & 0.024 & 0.000 & & & & & & \\
\hline
\end{tabular}


Table A1 - continued

\begin{tabular}{|c|c|c|c|c|c|c|c|c|c|c|c|}
\hline \multicolumn{6}{|c|}{ Initial Parameters } & \multicolumn{4}{|c|}{ Parameters at Period Minimum } & \multirow[b]{2}{*}{$\begin{array}{l}\text { gap } \\
\text { (hr) }\end{array}$} & \multirow[b]{2}{*}{ weight } \\
\hline $\begin{array}{c}M_{1} \\
\left(\mathrm{M}_{\odot}\right)\end{array}$ & $\begin{array}{c}M_{2} \\
\left(\mathrm{M}_{\odot}\right)\end{array}$ & $\begin{array}{c}P^{i} \\
(\mathrm{hr})\end{array}$ & $\begin{array}{c}t^{i} \\
(\mathrm{yr})\end{array}$ & $X_{c}^{i}$ & $M_{c}^{i} / M$ & $\begin{array}{l}P_{\min } \\
(\min )\end{array}$ & $\begin{array}{l}M_{2}^{\min } \\
\left(\mathrm{M}_{\odot}\right)\end{array}$ & $\begin{array}{c}\dot{M}_{\min } \\
\left(\mathrm{M}_{\odot} \mathrm{yr}^{-1}\right)\end{array}$ & $X_{s}^{\min }$ & & \\
\hline 0.8 & 0.6 & 4.2 & $0.0 \mathrm{E}+00$ & 0.685 & 0.000 & 77.1 & 0.068 & $2.6 \mathrm{E}-11$ & 0.683 & $2.64-3.03$ & $2.0 \mathrm{E}-02$ \\
\hline 0.8 & 0.8 & 5.4 & $0.0 \mathrm{E}+00$ & 0.685 & 0.000 & 75.8 & 0.068 & $2.4 \mathrm{E}-11$ & 0.682 & $2.58-3.02$ & $1.0 \mathrm{E}-02$ \\
\hline 0.8 & 0.8 & 5.6 & $3.9 \mathrm{E}+09$ & 0.560 & 0.000 & 75.0 & 0.068 & $2.4 \mathrm{E}-11$ & 0.663 & $2.45-2.89$ & $8.9 \mathrm{E}-03$ \\
\hline 0.8 & 0.8 & 5.9 & $8.0 \mathrm{E}+09$ & 0.436 & 0.000 & 75.3 & 0.064 & $2.4 \mathrm{E}-11$ & 0.642 & $2.15-2.50$ & $1.5 \mathrm{E}-03$ \\
\hline 0.8 & 0.9 & 6.0 & $0.0 \mathrm{E}+00$ & 0.685 & 0.000 & 76.0 & 0.068 & $2.4 \mathrm{E}-11$ & 0.682 & $2.59-3.02$ & $7.9 \mathrm{E}-03$ \\
\hline 0.8 & 0.9 & 6.3 & $2.7 \mathrm{E}+09$ & 0.552 & 0.000 & 75.3 & 0.064 & $2.2 \mathrm{E}-11$ & 0.660 & $2.44-2.88$ & $4.6 \mathrm{E}-03$ \\
\hline 0.8 & 0.9 & 6.7 & $5.1 \mathrm{E}+09$ & 0.433 & 0.000 & 75.0 & 0.064 & $2.5 \mathrm{E}-11$ & 0.638 & $2.08-2.45$ & $9.8 \mathrm{E}-04$ \\
\hline 0.8 & 0.9 & 7.1 & $7.7 \mathrm{E}+09$ & 0.310 & 0.000 & 72.4 & 0.064 & $2.3 \mathrm{E}-11$ & 0.610 & $1.67-1.87$ & $7.6 \mathrm{E}-04$ \\
\hline 0.8 & 0.9 & 8.4 & $1.2 \mathrm{E}+10$ & 0.093 & 0.000 & 61.1 & 0.050 & $2.4 \mathrm{E}-11$ & 0.399 & $1.19-1.43$ & $3.6 \mathrm{E}-04$ \\
\hline 0.8 & 1.0 & 6.8 & $0.0 \mathrm{E}+00$ & 0.685 & 0.000 & 76.6 & 0.069 & $2.8 \mathrm{E}-11$ & 0.682 & $2.55-3.02$ & $7.0 \mathrm{E}-03$ \\
\hline 0.8 & 1.0 & 7.1 & $1.5 \mathrm{E}+09$ & 0.567 & 0.000 & 75.3 & 0.068 & $2.7 \mathrm{E}-11$ & 0.661 & $2.43-2.88$ & $4.4 \mathrm{E}-03$ \\
\hline 0.8 & 1.0 & 7.5 & $3.2 \mathrm{E}+09$ & 0.444 & 0.000 & 73.8 & 0.063 & $2.3 \mathrm{E}-11$ & 0.637 & $2.08-2.44$ & $1.1 \mathrm{E}-03$ \\
\hline 0.8 & 1.0 & 8.0 & $4.6 \mathrm{E}+09$ & 0.327 & 0.000 & 69.5 & 0.075 & $2.8 \mathrm{E}-11$ & 0.590 & $1.35-1.56$ & $9.2 \mathrm{E}-04$ \\
\hline 0.8 & 1.0 & 9.3 & $7.1 \mathrm{E}+09$ & 0.101 & 0.000 & 66.1 & 0.042 & $1.5 \mathrm{E}-11$ & 0.461 & $1.48-1.59$ & $8.9 \mathrm{E}-04$ \\
\hline 0.8 & 1.0 & 11.2 & $9.0 \mathrm{E}+09$ & 0.000 & 0.000 & 53.8 & 0.029 & $1.3 \mathrm{E}-11$ & 0.255 & & $4.0 \mathrm{E}-04$ \\
\hline 0.8 & 1.0 & 13.6 & $1.0 \mathrm{E}+10$ & 0.000 & 0.036 & 23.2 & 0.068 & $7.2 \mathrm{E}-10$ & 0.104 & & $2.1 \mathrm{E}-04$ \\
\hline 0.8 & 1.0 & 15.9 & $1.1 \mathrm{E}+10$ & 0.000 & 0.063 & 10.4 & 0.110 & $1.4 \mathrm{E}-08$ & 0.010 & & $1.5 \mathrm{E}-04$ \\
\hline 0.8 & 1.0 & 19.0 & $1.1 \mathrm{E}+10$ & 0.000 & 0.093 & & & & & & \\
\hline 0.8 & 1.1 & 7.7 & $0.0 \mathrm{E}+00$ & 0.685 & 0.000 & 76.2 & 0.067 & $2.5 \mathrm{E}-11$ & 0.682 & $2.52-3.01$ & $7.5 \mathrm{E}-03$ \\
\hline 0.8 & 1.1 & 8.2 & $1.1 \mathrm{E}+09$ & 0.558 & 0.000 & 75.0 & 0.066 & $2.4 \mathrm{E}-11$ & 0.658 & $2.40-2.86$ & $4.0 \mathrm{E}-03$ \\
\hline 0.8 & 1.1 & 8.6 & $2.1 \mathrm{E}+09$ & 0.448 & 0.000 & 74.8 & 0.065 & $2.5 \mathrm{E}-11$ & 0.637 & $2.19-2.52$ & $8.9 \mathrm{E}-04$ \\
\hline 0.8 & 1.1 & 9.1 & $2.9 \mathrm{E}+09$ & 0.340 & 0.000 & 72.9 & 0.062 & $2.3 \mathrm{E}-11$ & 0.612 & $1.75-2.01$ & $8.1 \mathrm{E}-04$ \\
\hline 0.8 & 1.1 & 10.2 & $4.3 \mathrm{E}+09$ & 0.105 & 0.000 & 65.3 & 0.055 & $2.5 \mathrm{E}-11$ & 0.457 & $1.25-1.54$ & $8.6 \mathrm{E}-04$ \\
\hline 0.8 & 1.1 & 12.0 & $5.6 \mathrm{E}+09$ & 0.000 & 0.000 & 59.7 & 0.034 & $1.2 \mathrm{E}-11$ & 0.333 & & $6.2 \mathrm{E}-04$ \\
\hline 0.8 & 1.1 & 16.0 & $7.1 \mathrm{E}+09$ & 0.000 & 0.038 & 16.7 & 0.079 & $2.0 \mathrm{E}-09$ & 0.026 & & $4.2 \mathrm{E}-04$ \\
\hline 0.8 & 1.1 & 18.9 & $7.5 \mathrm{E}+09$ & 0.000 & 0.070 & & & & & & \\
\hline 0.8 & 1.2 & 8.8 & $0.0 \mathrm{E}+00$ & 0.685 & 0.000 & 76.4 & 0.071 & $2.9 \mathrm{E}-11$ & 0.682 & $2.53-3.01$ & $9.7 \mathrm{E}-03$ \\
\hline 0.8 & 1.2 & 9.7 & $1.4 \mathrm{E}+09$ & 0.567 & 0.000 & 74.3 & 0.065 & $2.6 \mathrm{E}-11$ & 0.635 & $2.01-2.38$ & $7.5 \mathrm{E}-03$ \\
\hline 0.8 & 1.2 & 11.0 & $2.7 \mathrm{E}+09$ & 0.447 & 0.000 & 70.1 & 0.052 & $1.8 \mathrm{E}-11$ & 0.551 & $1.32-1.40$ & $1.9 \mathrm{E}-03$ \\
\hline 0.8 & 1.2 & 12.5 & $3.7 \mathrm{E}+09$ & 0.329 & 0.000 & 63.2 & 0.042 & $1.6 \mathrm{E}-11$ & 0.416 & $1.88-2.23$ & $2.4 \mathrm{E}-03$ \\
\hline 0.8 & 1.2 & 15.5 & $5.2 \mathrm{E}+09$ & 0.105 & 0.000 & 46.7 & 0.049 & $4.8 \mathrm{E}-11$ & 0.175 & & $7.3 \mathrm{E}-04$ \\
\hline 0.8 & 1.2 & 16.3 & $5.4 \mathrm{E}+09$ & 0.047 & 0.000 & 28.6 & 0.067 & $4.0 \mathrm{E}-10$ & 0.041 & & $2.1 \mathrm{E}-04$ \\
\hline 0.8 & 1.2 & 17.0 & $5.6 \mathrm{E}+09$ & 0.010 & 0.000 & & & & & & \\
\hline 0.8 & 1.4 & 10.5 & $2.1 \mathrm{E}+07$ & 0.680 & 0.000 & 76.9 & 0.068 & $2.6 \mathrm{E}-11$ & 0.673 & $2.51-3.00$ & $5.7 \mathrm{E}-03$ \\
\hline 0.8 & 1.4 & 12.1 & $1.0 \mathrm{E}+09$ & 0.549 & 0.000 & 72.8 & 0.064 & $2.6 \mathrm{E}-11$ & 0.589 & $1.81-2.11$ & $4.2 \mathrm{E}-03$ \\
\hline 0.8 & 1.4 & 14.0 & $1.7 \mathrm{E}+09$ & 0.437 & 0.000 & 69.2 & 0.060 & $2.6 \mathrm{E}-11$ & 0.522 & $2.19-2.49$ & $1.2 \mathrm{E}-03$ \\
\hline 0.8 & 1.4 & 16.1 & $2.2 \mathrm{E}+09$ & 0.340 & 0.000 & 66.5 & 0.049 & $2.0 \mathrm{E}-11$ & 0.471 & $2.06-2.29$ & $8.7 \mathrm{E}-04$ \\
\hline 0.8 & 1.4 & 18.8 & $2.7 \mathrm{E}+09$ & 0.221 & 0.000 & 60.4 & 0.049 & $2.5 \mathrm{E}-11$ & 0.358 & $1.79-1.87$ & $5.5 \mathrm{E}-04$ \\
\hline 0.8 & 1.4 & 21.3 & $3.1 \mathrm{E}+09$ & 0.112 & 0.000 & 40.0 & 0.050 & $8.0 \mathrm{E}-11$ & 0.104 & & $3.2 \mathrm{E}-04$ \\
\hline 0.8 & 1.4 & 23.3 & $3.3 \mathrm{E}+09$ & 0.023 & 0.000 & & & & & & \\
\hline
\end{tabular}


Table A1 - continued

\begin{tabular}{|c|c|c|c|c|c|c|c|c|c|c|c|}
\hline \multicolumn{6}{|c|}{ Initial Parameters } & \multicolumn{4}{|c|}{ Parameters at Period Minimum } & \multirow[b]{2}{*}{$\begin{array}{l}\text { gap } \\
(\mathrm{hr})\end{array}$} & \multirow[b]{2}{*}{ weight } \\
\hline $\begin{array}{c}M_{1} \\
\left(\mathrm{M}_{\odot}\right)\end{array}$ & $\begin{array}{c}M_{2} \\
\left(\mathrm{M}_{\odot}\right)\end{array}$ & $\begin{array}{c}P^{i} \\
(\mathrm{hr})\end{array}$ & $\begin{array}{c}t^{i} \\
(\mathrm{yr})\end{array}$ & $X_{c}^{i}$ & $M_{c}^{i} / M$ & $\begin{array}{l}P_{\min } \\
(\min )\end{array}$ & $\begin{array}{l}M_{2}^{\min } \\
\left(\mathrm{M}_{\odot}\right)\end{array}$ & $\begin{array}{c}\dot{M}_{\min } \\
\left(\mathrm{M}_{\odot} \mathrm{yr}^{-1}\right)\end{array}$ & $X_{s}^{\min }$ & & \\
\hline 1.0 & 0.6 & 4.3 & $0.0 \mathrm{E}+00$ & 0.685 & 0.000 & 77.1 & 0.067 & $2.8 \mathrm{E}-11$ & 0.683 & $2.76-3.03$ & $1.3 \mathrm{E}-02$ \\
\hline 1.0 & 0.8 & 5.5 & $0.0 \mathrm{E}+00$ & 0.685 & 0.000 & 77.1 & 0.067 & $2.7 \mathrm{E}-11$ & 0.682 & $2.65-3.01$ & $6.4 \mathrm{E}-03$ \\
\hline 1.0 & 0.8 & 5.8 & $3.9 \mathrm{E}+09$ & 0.560 & 0.000 & 76.2 & 0.066 & $2.7 \mathrm{E}-11$ & 0.664 & $2.51-2.87$ & $5.7 \mathrm{E}-03$ \\
\hline 1.0 & 0.8 & 6.0 & $8.0 \mathrm{E}+09$ & 0.436 & 0.000 & 75.7 & 0.065 & $2.6 \mathrm{E}-11$ & 0.643 & $2.29-2.62$ & $1.1 \mathrm{E}-03$ \\
\hline 1.0 & 0.9 & 6.1 & $0.0 \mathrm{E}+00$ & 0.685 & 0.000 & 77.1 & 0.066 & $2.5 \mathrm{E}-11$ & 0.682 & $2.66-3.01$ & $4.5 \mathrm{E}-03$ \\
\hline 1.0 & 0.9 & 6.4 & $2.7 \mathrm{E}+09$ & 0.552 & 0.000 & 75.8 & 0.066 & $2.6 \mathrm{E}-11$ & 0.661 & $2.49-2.84$ & $2.7 \mathrm{E}-03$ \\
\hline 1.0 & 0.9 & 6.8 & $5.1 \mathrm{E}+09$ & 0.433 & 0.000 & 75.8 & 0.063 & $2.8 \mathrm{E}-11$ & 0.639 & $2.22-2.56$ & $7.6 \mathrm{E}-04$ \\
\hline 1.0 & 0.9 & 7.2 & $7.7 \mathrm{E}+09$ & 0.310 & 0.000 & 73.3 & 0.063 & $2.6 \mathrm{E}-11$ & 0.612 & $1.69-1.88$ & $5.6 \mathrm{E}-04$ \\
\hline 1.0 & 0.9 & 8.5 & $1.2 \mathrm{E}+10$ & 0.093 & 0.000 & 64.6 & 0.033 & $1.1 \mathrm{E}-11$ & 0.394 & & $2.5 \mathrm{E}-04$ \\
\hline 1.0 & 1.0 & 6.9 & $0.0 \mathrm{E}+00$ & 0.685 & 0.000 & 77.8 & 0.070 & $3.4 \mathrm{E}-11$ & 0.682 & $2.67-3.01$ & $4.0 \mathrm{E}-03$ \\
\hline 1.0 & 1.0 & 7.3 & $1.5 \mathrm{E}+09$ & 0.567 & 0.000 & 75.5 & 0.067 & $2.7 \mathrm{E}-11$ & 0.662 & $2.50-2.88$ & $2.7 \mathrm{E}-03$ \\
\hline 1.0 & 1.0 & 7.7 & $3.2 \mathrm{E}+09$ & 0.444 & 0.000 & 75.0 & 0.066 & $2.8 \mathrm{E}-11$ & 0.638 & $2.16-2.50$ & $7.5 \mathrm{E}-04$ \\
\hline 1.0 & 1.0 & 8.2 & $4.6 \mathrm{E}+09$ & 0.327 & 0.000 & 72.9 & 0.061 & $2.5 \mathrm{E}-11$ & 0.595 & $1.42-1.57$ & $7.7 \mathrm{E}-04$ \\
\hline 1.0 & 1.0 & 9.5 & $7.1 \mathrm{E}+09$ & 0.101 & 0.000 & 67.8 & 0.052 & $2.4 \mathrm{E}-11$ & 0.483 & $1.56-1.59$ & $7.1 \mathrm{E}-04$ \\
\hline 1.0 & 1.0 & 11.4 & $9.0 \mathrm{E}+09$ & 0.000 & 0.000 & 53.9 & 0.058 & $8.9 \mathrm{E}-11$ & 0.316 & & $3.5 \mathrm{E}-04$ \\
\hline 1.0 & 1.0 & 13.9 & $1.0 \mathrm{E}+10$ & 0.000 & 0.034 & 22.0 & 0.063 & $8.9 \mathrm{E}-10$ & 0.034 & & $1.7 \mathrm{E}-04$ \\
\hline 1.0 & 1.0 & 16.3 & $1.1 \mathrm{E}+10$ & 0.000 & 0.060 & 7.6 & 0.125 & $4.9 \mathrm{E}-08$ & 0.000 & & $9.4 \mathrm{E}-05$ \\
\hline 1.0 & 1.0 & 19.5 & $1.2 \mathrm{E}+10$ & 0.000 & 0.093 & & & & & & \\
\hline 1.0 & 1.1 & 7.9 & $0.0 \mathrm{E}+00$ & 0.685 & 0.000 & 77.1 & 0.067 & $2.6 \mathrm{E}-11$ & 0.682 & $2.59-3.02$ & $4.4 \mathrm{E}-03$ \\
\hline 1.0 & 1.1 & 8.4 & $1.1 \mathrm{E}+09$ & 0.558 & 0.000 & 75.8 & 0.065 & $2.6 \mathrm{E}-11$ & 0.659 & $2.49-2.83$ & $2.3 \mathrm{E}-03$ \\
\hline 1.0 & 1.1 & 8.8 & $2.1 \mathrm{E}+09$ & 0.448 & 0.000 & 75.5 & 0.064 & $2.7 \mathrm{E}-11$ & 0.637 & $2.25-2.53$ & $6.6 \mathrm{E}-04$ \\
\hline 1.0 & 1.1 & 9.3 & $2.9 \mathrm{E}+09$ & 0.340 & 0.000 & 73.6 & 0.062 & $2.5 \mathrm{E}-11$ & 0.615 & $1.84-2.05$ & $6.6 \mathrm{E}-04$ \\
\hline 1.0 & 1.1 & 10.4 & $4.3 \mathrm{E}+09$ & 0.105 & 0.000 & 70.3 & 0.060 & $2.9 \mathrm{E}-11$ & 0.534 & $1.34-1.42$ & $6.2 \mathrm{E}-04$ \\
\hline 1.0 & 1.1 & 12.3 & $5.6 \mathrm{E}+09$ & 0.000 & 0.000 & 60.0 & 0.032 & $1.3 \mathrm{E}-11$ & 0.321 & & $4.7 \mathrm{E}-04$ \\
\hline 1.0 & 1.1 & 16.4 & $7.1 \mathrm{E}+09$ & 0.000 & 0.038 & 15.6 & 0.081 & $3.2 \mathrm{E}-09$ & 0.032 & & $2.5 \mathrm{E}-04$ \\
\hline 1.0 & 1.1 & 19.4 & $7.5 \mathrm{E}+09$ & 0.000 & 0.072 & & & & & & \\
\hline 1.0 & 1.2 & 9.0 & $0.0 \mathrm{E}+00$ & 0.685 & 0.000 & 78.2 & 0.067 & $2.6 \mathrm{E}-11$ & 0.682 & $2.58-3.05$ & $6.0 \mathrm{E}-03$ \\
\hline 1.0 & 1.2 & 10.0 & $1.4 \mathrm{E}+09$ & 0.567 & 0.000 & 74.3 & 0.065 & $2.8 \mathrm{E}-11$ & 0.636 & $2.08-2.40$ & $4.3 \mathrm{E}-03$ \\
\hline 1.0 & 1.2 & 11.3 & $2.7 \mathrm{E}+09$ & 0.447 & 0.000 & 70.6 & 0.069 & $3.3 \mathrm{E}-11$ & 0.562 & $1.39-1.50$ & $1.1 \mathrm{E}-03$ \\
\hline 1.0 & 1.2 & 12.8 & $3.7 \mathrm{E}+09$ & 0.329 & 0.000 & 63.2 & 0.051 & $2.5 \mathrm{E}-11$ & 0.417 & $1.92-2.11$ & $1.4 \mathrm{E}-03$ \\
\hline 1.0 & 1.2 & 15.9 & $5.2 \mathrm{E}+09$ & 0.105 & 0.000 & 43.5 & 0.048 & $4.4 \mathrm{E}-11$ & 0.152 & & $6.5 \mathrm{E}-04$ \\
\hline 1.0 & 1.2 & 16.7 & $5.4 \mathrm{E}+09$ & 0.047 & 0.000 & 23.1 & 0.074 & $9.6 \mathrm{E}-10$ & 0.031 & & $1.6 \mathrm{E}-04$ \\
\hline 1.0 & 1.2 & 17.5 & $5.6 \mathrm{E}+09$ & 0.010 & 0.000 & & & & & & \\
\hline 1.0 & 1.4 & 10.8 & $2.1 \mathrm{E}+07$ & 0.680 & 0.000 & 76.2 & 0.065 & $2.8 \mathrm{E}-11$ & 0.637 & $2.43-2.84$ & $3.8 \mathrm{E}-03$ \\
\hline 1.0 & 1.4 & 12.5 & $1.0 \mathrm{E}+09$ & 0.548 & 0.000 & 71.9 & 0.061 & $2.7 \mathrm{E}-11$ & 0.557 & $2.19-2.52$ & $2.4 \mathrm{E}-03$ \\
\hline 1.0 & 1.4 & 14.4 & $1.7 \mathrm{E}+09$ & 0.436 & 0.000 & 68.7 & 0.057 & $2.8 \mathrm{E}-11$ & 0.500 & $2.08-2.34$ & $6.9 \mathrm{E}-04$ \\
\hline 1.0 & 1.4 & 16.5 & $2.2 \mathrm{E}+09$ & 0.340 & 0.000 & 65.6 & 0.052 & $2.6 \mathrm{E}-11$ & 0.436 & $1.94-2.11$ & $5.2 \mathrm{E}-04$ \\
\hline 1.0 & 1.4 & 19.3 & $2.7 \mathrm{E}+09$ & 0.221 & 0.000 & 54.3 & 0.054 & $4.2 \mathrm{E}-11$ & 0.255 & $1.66-1.67$ & $4.4 \mathrm{E}-04$ \\
\hline 1.0 & 1.4 & 21.9 & $3.1 \mathrm{E}+09$ & 0.112 & 0.000 & 38.5 & 0.052 & $1.1 \mathrm{E}-10$ & 0.090 & & $2.4 \mathrm{E}-04$ \\
\hline 1.0 & 1.4 & 23.9 & $3.3 \mathrm{E}+09$ & 0.023 & 0.000 & & & & & & \\
\hline
\end{tabular}

Note. - $M_{1}$ : mass of white dwarf; $M_{2}$ : initial mass of secondary; $P^{i}$ : initial orbital period; $t^{i}$ : age at beginning of mass transfer; $X_{c}^{i}$ : initial central hydrogen mass fraction; $M_{c}^{i} / M$ : initial fractional mass of the H-exhausted core; $P_{\text {min }}$ : minimum period; $M_{2}^{\text {min }}$ : secondary mass at period minimum, $\dot{M}_{\min }$ : mass-transfer rate at period minimum; $X_{s}^{\text {min }}$ : surface $\mathrm{H}$ abundance at period minimum; gap: period gap; $M_{2}^{\text {gap }}$ : secondary mass at beginning of period gap; relative weight of sequence in model (1). 